\title{
High order finite volume WENO schemes for the Euler equations under gravitational fields
}

\author{
Gang $\mathrm{Li}^{1}$ and Yulong Xing ${ }^{2}$
}

\begin{abstract}
Euler equations with gravitational source terms are used to model many astrophysical and atmospheric phenomena. This system admits hydrostatic balance where the flux produced by the pressure is exactly canceled by the gravitational source term, and two commonly seen equilibria are the isothermal and polytropic hydrostatic solutions. Exact preservation of these equilibria is desirable as many practical problems are small perturbations of such balance. High order finite difference weighted essentially non-oscillatory (WENO) schemes have been proposed in [22], but only for the isothermal equilibrium state. In this paper, we design high order well-balanced finite volume WENO schemes, which can preserve not only the isothermal equilibrium but also the polytropic hydrostatic balance state exactly, and maintain genuine high order accuracy for general solutions. The well-balanced property is obtained by novel source term reformulation and discretization, combined with well-balanced numerical fluxes. Extensive one- and two-dimensional simulations are performed to verify well-balanced property, high order accuracy, as well as good resolution for smooth and discontinuous solutions.
\end{abstract}

Keywords: Finite volume schemes; Euler equations; WENO schemes; Well-balanced property; High order accuracy; Gravitational field

\footnotetext{
${ }^{1}$ School of mathematics and statistics, Qingdao University, Qingdao, Shandong 266071, P.R. China. Email: gangli1978@163.com.

${ }^{2}$ Corresponding author. Department of Mathematics, University of California Riverside, Riverside, CA 92521, U.S.A. E-mail: xingy@ucr.edu. Tel.: (951) 827-7347. Fax: (951) 827-7314.

1

(c) 2016. This manuscript version is made available under the Elsevier user license http://www.elsevier.com/open-access/userlicense/1.0/
} 


\section{Introduction}

Euler equations with gravitational source terms are widely used to model many interesting physical phenomena in the astrophysical and atmospheric science. These equations governing the conservation of mass, momentum and energy, coupled with source terms due to the gravitational field, are given by

$$
\begin{aligned}
& \rho_{t}+\nabla \cdot(\rho \mathbf{u})=0, \\
& (\rho \mathbf{u})_{t}+\nabla \cdot\left(\rho \mathbf{u} \otimes \mathbf{u}+p \mathbf{I}_{d}\right)=-\rho \nabla \phi, \\
& E_{t}+\nabla \cdot((E+p) \mathbf{u})=-\rho \mathbf{u} \cdot \nabla \phi,
\end{aligned}
$$

where $\mathbf{x} \in \mathcal{R}^{d}(d=1,2,3)$ is the spatial variable, $\rho$ denotes the fluid density, $\mathbf{u}$ is the velocity, $p$ represents the pressure, and $E=\frac{1}{2} \rho\|\mathbf{u}\|^{2}+\rho e$ ( $e$ is internal energy) is the nongravitational energy which includes the kinetic and internal energy of the fluid. $\phi=\phi(\mathbf{x})$ is the time independent gravitational potential. The operators $\nabla, \nabla \cdot$ and $\otimes$ are the gradient, divergence and tensor product in $\mathcal{R}^{d}$, respectively, and $\mathbf{I}_{d}$ stands for the identity matrix. To close the system, the pressure $p$ is linked to the density and the interval energy through an equation of state denoted by $p=p(\rho, e)$. The ideal gas law for the equation of state takes the form of

$$
p=(\gamma-1) \rho e=(\gamma-1)\left(E-\rho\|\mathbf{u}\|^{2} / 2\right)
$$

where $\gamma$ is the ratio of specific heats.

In one spatial dimension, the Euler equations (1.1) take the form of

$$
\begin{aligned}
& \rho_{t}+(\rho u)_{x}=0 \\
& (\rho u)_{t}+\left(\rho u^{2}+p\right)_{x}=-\rho \phi_{x} \\
& E_{t}+((E+p) u)_{x}=-\rho u \phi_{x}
\end{aligned}
$$

with $u$ being the velocity. Such model belongs to the general class of hyperbolic conservation laws with source terms, often referred as hyperbolic balance laws, which takes the general form of

$$
U_{t}+f(U)_{x}=S(U, \phi)
$$


where $U$ is the solution vector with the corresponding flux $f(U)$, and $S(U, \phi)$ is the source term. The balance law usually admits non-trivial steady state solutions, in which the source term is exactly balanced by the flux gradient.

The Euler equations (1.3) under the static gravitation potential admit the hydrostatic stationary solution, also called mechanical equilibrium, where the velocity is zero and the external forces such as gravity are balanced by the pressure gradient force:

$$
\rho=\rho(x), \quad u=0, \quad p_{x}=-\rho \phi_{x} .
$$

Two important special steady state arising in the applications are the constant temperature (isothermal) [22] and polytropic hydrostatic equilibrium states [9].

If the hydrostatic state is isothermal, we have $T(x) \equiv T_{0}=$ const with $T(x)$ being the temperature. For an ideal gas satisfying

$$
p(x)=\rho(x) R T(x),
$$

where $R$ is the gas constant, integrating the steady state solution (1.5) yields

$$
\rho=\frac{p_{0}}{R T(x)} \exp \left(-\int_{x_{0}}^{x} \frac{\phi_{x}(s)}{R T(s)} d s\right), \quad u=0, \quad p=p_{0} \exp \left(-\int_{x_{0}}^{x} \frac{\phi_{x}(s)}{R T(s)} d s\right),
$$

where $p_{0}$ is the initial pressure at some reference position $x_{0}$. Under the isothermal assumption, the equilibrium correspondingly becomes

$$
\rho=\rho_{0} \exp \left(-\frac{\phi}{R T_{0}}\right), \quad u=0, \quad p=p_{0} \exp \left(-\frac{\phi}{R T_{0}}\right)
$$

with $p_{0}=\rho_{0} R T_{0}$.

The other polytropic hydrostatic equilibrium is characterized by

$$
p=K \rho^{\nu},
$$

which will lead to the form of

$$
\rho=\left(\frac{\nu-1}{K \nu}(C-\phi)\right)^{\frac{1}{\nu-1}}, \quad u=0, \quad p=\frac{1}{K^{\frac{1}{\nu-1}}}\left(\frac{\nu-1}{\nu}(C-\phi)\right)^{\frac{\nu}{\nu-1}}
$$


where $C, K$ and $\nu$ are all constants. A special case of $\nu$ is the ratio of specific heats $\gamma$.

The simplest encountered gravity is the linear gravitational potential field with $\phi_{x}=g$, and the corresponding isothermal and polytropic hydrostatic balances take the form of

$$
\rho=\rho_{0} \exp \left(\frac{-g \rho_{0} x}{p_{0}}\right), \quad u=0, \quad p=p_{0} \exp \left(\frac{-g \rho_{0} x}{p_{0}}\right)
$$

and

$$
p=p_{0}^{\frac{1}{\nu-1}}\left(p_{0}-\frac{\nu-1}{\nu} g \rho_{0} x\right)^{\frac{\nu}{\nu-1}}, \quad u=0, \quad \rho=\rho_{0}\left(\frac{p}{p_{0}}\right)^{\frac{1}{\nu}} .
$$

Many physical applications of the Euler equations under a gravitational field involve nearly steady state flows which are small perturbation of the hydrostatic equilibrium states. It is important to correctly capture the effect of gravitational force in these simulations, especially if a long-time integration is involved, for example in modeling star and galaxy formation. Improper treatment of the gravitational force can lead to a solution which either oscillates around the equilibrium, or deviates from the equilibrium after a long time run. The problem can be resolved if the mesh size is extremely refined, which may not be feasible in some applications. To save the computational cost, well-balanced schemes, which preserve exactly these steady state solutions up to machine accuracy, are specially designed to ensure accurate simulations and exhibit essential stability properties on relatively coarse meshes. Another prototypical example considered extensively in the literature for hyperbolic balance laws is the shallow water equations over a non-flat bottom topology. Many well-balanced methods for the shallow water equations were designed in the past decade, see, e.g. [2, 7,1 , $11,15,25,20,24,23]$ and the references therein.

Well-balanced numerical schemes for the Euler equations under a gravitational field have attracted many attention recently. LeVeque and Bale [12] have extended the quasi-steady wave-propagation methods to the Euler equations. Later, finite volume well-balanced discretizations has been proposed by Botta et al. [3] for the nearly hydrostatic flows in the numerical weather prediction. Gas-kinetic schemes have been extended by Xu and his collaborators $[19,26,14]$ to the multidimensional gas dynamic equations to develop well-balanced 
numerical methods, where the gravitational potential was modeled as a piecewise step function with a potential jump at the cell interface. High order finite difference well-balanced schemes for the isothermal equilibrium only are introduced in [22] by Xing and Shu. Second order finite volume well-balanced schemes for the isentropic hydrostatic equilibrium are proposed by Käppeli and Mishra [9]. They have extended their results to general hydrostatic equilibrium without any assumption of a thermal equilibrium in [10], by introducing a local second-order hydrostatic equilibrium preserving pressure reconstruction. Chertock et al. [5] designed a second-order well-balanced central-upwind scheme for the Euler equations of gas dynamics with gravitation. Chandrashekar and Klingenberg [4] extend the approach in [22] and provide a well-balanced second order Godunov-type method, which can preserve both isothermal and polytropic stationary solutions exactly. We applied the technique in [22] to design a high order well-balanced discontinuous Galerkin method for the isothermal model [13]. More recently, Ghosh et al. have studied the applications of well-balanced methods for the numerical simulation of atmospheric flows [6]. By extending the result in [22], they designed well-balanced finite difference WENO and compact-reconstruction WENO methods for a more general form of the hydrostatic balance, including the isothermal equilibrium and other stratified atmosphere encountered in the applications.

In this paper, we plan to extend the well-balanced technique in [22] from several aspects. First, we propose to design high order well-balanced finite volume weighted non-oscillatory (WENO) schemes. Finite volume schemes represent the underlying physics in a natural way, and are very popular for solving hyperbolic conservation laws. They are widely used in the physical applications. Second, the well-balanced technique in [22] is only designed for the isothermal equilibrium. A nice second order generalization to both isothermal and polytropic cases is provided in [4], which may not be extended to high order version in a straightforward way. Here, we provide a different approach to apply high order well-balanced technique on the polytropic equilibrium. Similarly as in [22], we first rewrite the source terms in an equivalent special form using the corresponding hydrostatic equilibrium (1.7) or (1.8). 
They are then discretized to be both high order accurate for general solutions and exactly well-balanced with the pressure gradient at the equilibrium state. The extra computational cost to obtain the well-balanced property can be negligible.

This paper is organized as follows. In Section 2, we first present the novel one-dimensional high order well-balanced WENO schemes, which can preserve both the isothermal and polytropoc hydrostatic balance solutions exactly. Section 3 contains extensive numerical simulation results to demonstrate the behavior of our well-balanced WENO schemes for one-dimensional Euler equations under a gravitational field. We then extend the proposed well-balanced schemes to multi-dimensional cases in Section 4, and present some numerical results in Section 5. In Section 6, some conclusion remarks are provided.

\section{One-dimensional well-balanced WENO schemes}

In this section, we present high order well-balanced finite volume WENO schemes for the Euler equations under gravitational fields. We begin with the isothermal case, and then extend the idea to the polytropic case. We will confine our discussion to one-dimensional problem only in this section, and discuss the generalization to multi-dimensional case in Section 4.

\subsection{Notations and WENO reconstruction}

We first introduce some notations and the standard WENO reconstruction which will be used later. In one spatial dimension, the computational domain is divided into cells $I_{j}=$ $\left[x_{j-1 / 2}, x_{j+1 / 2}\right]$. We denote the size of the $j$-th cell by $\Delta x_{j}$ and the maximum mesh size by

$\Delta x=\max _{j} \triangle x_{j}$. The usual notation $\{u\}=\frac{1}{2}\left(u^{+}+u^{-}\right)$is used to represent the arithmetic average of the function $u$ at the element interfaces.

In a finite volume scheme for the equations (1.4), our computational variables are $\bar{U}_{j}(t)$, which approximate the cell averages $\bar{U}\left(x_{j}, t\right)=\frac{1}{\Delta x_{j}} \int_{I_{j}} U(x, t) d x$. The conservative WENO 
scheme is given by

$$
\frac{d}{d t} \bar{U}_{j}(t)+\frac{1}{\Delta x_{j}}\left(\hat{f}_{j+\frac{1}{2}}-\hat{f}_{j-\frac{1}{2}}\right)=\frac{1}{\Delta x_{j}} \int_{I_{j}} S(U, \phi) d x,
$$

with $\hat{f}_{j+1 / 2}=F\left(U_{j+1 / 2}^{-}, U_{j+1 / 2}^{+}\right)$being the numerical flux. The simplest numerical flux is the well-known Lax-Friedrichs flux

$$
F(a, b)=\frac{1}{2}(f(a)+f(b)-\alpha(b-a))
$$

where $\alpha=\max (|u|+\sqrt{\gamma p / \rho})$ and the maximum is taken over the whole domain. $U_{j+1 / 2}^{-}$ and $U_{j+1 / 2}^{+}$are the high order pointwise approximations to $U\left(x_{j+1 / 2}, t\right)$ from left and right, respectively. They are computed through the neighboring cell average values by a high order WENO reconstruction procedure. Basically, for a $(2 k-1)$-th order WENO scheme, we first compute $k$ reconstructed boundary values $U_{j+1 / 2}^{(k), \pm}$ corresponding to different candidate stencils. Then by providing each value a weight which indicates the smoothness of the corresponding stencil, we define the $(2 k-1)$-th order WENO reconstruction $U_{j+1 / 2}^{ \pm}$as a convex combination of all these $k$ reconstructed values. Eventually, the WENO reconstruction can be written out as:

$$
U_{j+\frac{1}{2}}^{+}=\sum_{r=-k+1}^{k} w_{r} \bar{U}_{j+r}, \quad U_{j+\frac{1}{2}}^{-}=\sum_{r=-k}^{k-1} \tilde{w}_{r} \bar{U}_{j+r} .
$$

where $k=3$ for the fifth order WENO approximation and the coefficients $w_{r}$ and $\tilde{w}_{r}$ depend nonlinearly on the smoothness indicators involving the cell average $\bar{U}$. For hyperbolic systems of conservation laws, we usually apply the local characteristic decomposition procedure, which is more robust than a component by component version. The complete algorithm can be found in $[8,16]$.

For the temporal discretization, high order total variation diminishing (TVD) RungeKutta methods [17] can be used. In the numerical section of this paper, we apply the third 
order Runge-Kutta methods:

$$
\begin{aligned}
U^{(1)} & =U^{n}+\Delta t \mathcal{F}\left(U^{n}\right) \\
U^{(2)} & =\frac{3}{4} U^{n}+\frac{1}{4}\left(U^{(1)}+\Delta t \mathcal{F}\left(U^{(1)}\right)\right) \\
U^{n+1} & =\frac{1}{3} U^{n}+\frac{2}{3}\left(U^{(2)}+\Delta t \mathcal{F}\left(U^{(2)}\right)\right),
\end{aligned}
$$

with $\mathcal{F}(U)$ being the spatial operator.

\subsection{Well-balanced schemes for the isothermal equilibrium}

In this subsection, we will present well-balanced finite volume WENO schemes for the isothermal equilibrium (1.8). Following the approach in our finite difference work [22], we utilize the structure of the equilibrium (1.8) and first reformulate the original governing equations as

$$
\begin{aligned}
& \rho_{t}+(\rho u)_{x}=0, \\
& (\rho u)_{t}+\left(\rho u^{2}+p\right)_{x}=\frac{p_{0}}{\rho_{0}} \rho \exp \left(\frac{\phi}{R T_{0}}\right)\left(\exp \left(-\frac{\phi}{R T_{0}}\right)\right)_{x}, \\
& E_{t}+((E+p) u)_{x}=-\rho u \phi_{x},
\end{aligned}
$$

where $-\rho \phi_{x}$ in the second equation is replaced by $\frac{p_{0}}{\rho_{0}} \rho \exp \left(\frac{\phi}{R T_{0}}\right)\left(\exp \left(-\frac{\phi}{R T_{0}}\right)\right)_{x}$. By writing in this special form, we hope to create the derivative term in the source term, which can be treated in the similar way as the flux term at the steady state (1.8) to achieve the wellbalanced property. It is not necessary to change the source term in the last equation, since the well-balanced property for this equation can be easily obtained when $u=0$ at the steady state.

The standard WENO schemes as outlined in (2.1) do not have the well-balanced property automatically. To preserve the steady state solution (1.8), we need to introduce a nonstandard approximation to the integration of the second source term, denoted by $S^{[2]}$. For ease of presentation, we introduce the following notation

$$
d(x)=\exp \left(-\frac{\phi}{R T_{0}}\right),
$$


and the isothermal steady state solution (1.8) then becomes

$$
\rho=\rho_{0} d(x), \quad u=0, \quad p=p_{0} d(x) .
$$

The source term $S^{[2]}$ can be rewritten as $\frac{p_{0}}{\rho_{0}} \frac{\rho}{d} d_{x}$, and we can decompose its integral as

$$
\begin{aligned}
\frac{1}{\Delta x} \int_{I_{j}} S^{[2]} \mathrm{d} x & =\frac{1}{\Delta x} \frac{p_{0}}{\rho_{0}} \int_{I_{j}} \frac{\rho}{d} d_{x} \mathrm{~d} x \\
& =\frac{1}{\Delta x} \frac{p_{0}}{\rho_{0}} \int_{I_{j}}\left(\frac{\rho}{d}-\frac{\bar{\rho}_{j}}{\bar{d}_{j}}+\frac{\bar{\rho}_{j}}{\bar{d}_{j}}\right) d_{x} \mathrm{~d} x \\
& =\frac{1}{\Delta x} \frac{p_{0}}{\rho_{0}} \frac{\bar{\rho}_{j}}{\bar{d}_{j}}\left(d_{j+1 / 2}-d_{j-1 / 2}\right)+\frac{1}{\Delta x} \frac{p_{0}}{\rho_{0}} \int_{I_{j}}\left(\frac{\rho}{d}-\frac{\bar{\rho}_{j}}{\bar{d}_{j}}\right) d_{x} \mathrm{~d} x .
\end{aligned}
$$

Our numerical approximation to it takes the following form

$$
\frac{1}{\Delta x} \int_{I_{j}} S^{[2]} \mathrm{d} x \approx \frac{1}{\Delta x} \frac{p_{0}}{\rho_{0}} \frac{\bar{\rho}_{j}}{\bar{d}_{j}}\left(\left\{d_{j+1 / 2}\right\}-\left\{d_{j-1 / 2}\right\}\right)+\frac{1}{\Delta x} \frac{p_{0}}{\rho_{0}} \int_{I_{j}}\left(\frac{\rho}{d}-\frac{\bar{\rho}_{j}}{\bar{d}_{j}}\right) d_{x} \mathrm{~d} x,
$$

where $d_{j+1 / 2}$ is replaced by $\left\{d_{j+1 / 2}\right\}=\frac{1}{2}\left(d_{j+1 / 2}^{+}+d_{j+1 / 2}^{-}\right)$, and the integration in the last term can be obtained by the standard Gaussian quadrature rule. To achieve well-balanced property, the boundary terms $d_{j+1 / 2}^{ \pm}$are computed by applying the WENO reconstruction on the cell averages $\bar{d}_{j}$. For the integration of the source term in the third equation, we can apply the standard Gaussian quadrature rule to evaluate it.

The last piece in designing well-balanced WENO schemes is to slightly modify the numerical flux $\hat{f}_{j+1 / 2}$. The term $\alpha(b-a)$ in the Lax-Friedrichs flux (2.2) contributes to the numerical viscosity term, which is essential for nonlinear conservation laws. However they may destroy the well-balanced property at the steady state (1.8). Here, we propose to modify it as

$$
\hat{f}_{j+1 / 2}=\frac{1}{2}\left[f\left(U_{j+1 / 2}^{-}\right)+f\left(U_{j+1 / 2}^{+}\right)-\alpha^{\prime}\left(\frac{U_{j+1 / 2}^{+}}{d_{j+1 / 2}^{+}}-\frac{U_{j+1 / 2}^{-}}{d_{j+1 / 2}^{-}}\right)\right],
$$

where the coefficient $\alpha^{\prime}$ is defined as

$$
\alpha^{\prime}=\alpha \max _{j} \bar{d}_{j}
$$

to maintain enough artificial numerical viscosity. This modification does not affect the accuracy, but at the steady state (2.6) where the term $U / d$ becomes a constant, the effect of 
these viscosity terms disappears. The numerical flux now reduces to a simple form

$$
\hat{f}_{j+1 / 2}=\frac{1}{2}\left[f\left(U_{j+1 / 2}^{-}\right)+f\left(U_{j+1 / 2}^{+}\right)\right] .
$$

All these together lead to high order well-balanced WENO schemes for the Euler equations.

Proposition 1. For the Euler equations under the static gravitational potential field, reformulated in the form of (2.5), the semi-discrete WENO schemes (2.1), combined with the source term approximation (2.8) and the modified numerical fluxes (2.9), are well-balanced for the isothermal steady state solution (1.8).

Proof. At the steady state (1.8), we have

$$
\bar{\rho}_{j}=\rho_{0} \bar{d}_{j}, \quad \bar{u}_{j}=0, \quad \bar{p}_{j}=p_{0} \bar{d}_{j}
$$

Easy to observe that the well-balanced property holds for the first and third equations, as both the flux and source term approximations in these equations become zero. For the momentum equation, we have $\bar{\rho}_{j} / \bar{d}_{j}=\rho(x) / d(x)=\rho_{0}$, and the source term approximation (2.8) becomes

$$
\frac{1}{\Delta x} \int_{I_{j}} S^{[2]} \mathrm{d} x \approx \frac{1}{\Delta x} p_{0}\left(\left\{d_{j+1 / 2}\right\}-\left\{d_{j-1 / 2}\right\}\right) .
$$

Since $u=0$, the second flux term $f^{[2]}(U)=\rho u^{2}+p$ reduces to $p$. Its numerical approximation takes the form of

$$
\frac{1}{\Delta x}\left(\hat{f}_{j+1 / 2}^{[2]}-\hat{f}_{j+1 / 2}^{[2]}\right)=\frac{1}{\Delta x} p_{0}\left(\left\{d_{j+1 / 2}\right\}-\left\{d_{j-1 / 2}\right\}\right)
$$

where (2.11) is used. We can conclude that the flux and source term approximations balance exactly each other, which leads to the well-balanced property of our schemes.

We complete this subsection with some remarks.

Remark 1. The well-balanced finite volume WENO schemes constructed above are very similar to the well-balanced discontinuous Galerkin methods in [13], especially in the wellbalanced flux and the source term approximation. This is expected, due to the connection 
between finite volume schemes and discontinuous Galerkin methods. As commented in [13],

the choice of $\bar{\rho} / \bar{b}$ in (2.8) is not unique, and can be replace by any other term that can recover constant $\rho_{0}$ at the steady state (2.6).

Remark 2. The well-balanced finite volume schemes can also be viewed as the generalization of the well-balanced schemes designed for the shallow water equations in [21]. But unlike the shallow water case, we don't need to use the solution $U$ to compute the coefficients of the WENO reconstruction for $\bar{d}_{j}$, therefore our source term approximation is more nature in this sense.

Remark 3. In some examples, we may know the solutions converge to the isothermal equilibrium (2.6), but the constant $T_{0}$ is not explicitly given. In these cases, we could simply replace $T_{0}$ in (2.5) by the average of the temperature function $T$ computed at the current time level $t^{n}$.

\subsection{Well-balanced schemes for polytropic equilibrium}

Well-balanced WENO schemes have been designed for the isothermal steady state (1.8) in the previous subsection. In the subsection, we extend these schemes to the polytropic steady state (1.10). For ease of presentation, we introduce the following notations

$$
\rho^{e}(x)=\left(\frac{\nu-1}{K \nu}(C-\phi)\right)^{\frac{1}{\nu-1}}, \quad u=0, \quad p^{e}(x)=\frac{1}{K^{\frac{1}{\nu-1}}}\left(\frac{\nu-1}{\nu}(C-\phi)\right)^{\frac{\nu}{\nu-1}}
$$

for the equilibrium states. Note that these two functions only depend on the gravitation potential $\phi(x)$ and $\nu$, and are independent of the solutions $U$.

Similarly, we first take in consideration the targeting polytropic equilibrium state (2.14), and rewrite the governing equations (1.3) as

$$
\begin{aligned}
& \rho_{t}+(\rho u)_{x}=0 \\
& (\rho u)_{t}+\left(\rho u^{2}+p\right)_{x}=\frac{\rho}{\rho^{e}} p_{x}^{e} \\
& E_{t}+((E+p) u)_{x}=-\rho u \phi_{x},
\end{aligned}
$$


which is the analogy of the equations (2.5) for the isothermal steady state (1.8).

The semi-discrete well-balanced WENO schemes still take the form of (2.1), but with modified numerical fluxes and the source term approximations outlined below. Following the same technique as stated above, we decompose the integral of the source term in the second equation as

$$
\begin{aligned}
\frac{1}{\Delta x} \int_{I_{j}} S^{[2]} \mathrm{d} x & =\frac{1}{\Delta x} \int_{I_{j}} \frac{\rho}{\rho^{e}} p_{x}^{e} \mathrm{~d} x \\
& =\frac{1}{\Delta x} \int_{I_{j}}\left(\frac{\rho}{\rho^{e}}-\frac{\bar{\rho}_{j}}{\bar{\rho}_{j}^{e}}+\frac{\bar{\rho}_{j}}{\bar{\rho}_{j}^{e}}\right) p_{x}^{e} \mathrm{~d} x \\
& =\frac{1}{\Delta x} \frac{\bar{\rho}_{j}}{\bar{\rho}_{j}^{e}}\left(p_{j+1 / 2}^{e}-p_{j-1 / 2}^{e}\right)+\frac{1}{\Delta x} \int_{I_{j}}\left(\frac{\rho}{\rho^{e}}-\frac{\bar{\rho}_{j}}{\bar{\rho}_{j}^{e}}\right) p_{x}^{e} \mathrm{~d} x
\end{aligned}
$$

and approximate it by

$$
\frac{1}{\Delta x} \int_{I_{j}} S^{[2]} \mathrm{d} x \approx \frac{1}{\Delta x} \frac{\bar{\rho}_{j}}{\bar{\rho}_{j}^{e}}\left(\left\{p_{j+1 / 2}^{e}\right\}-\left\{p_{j-1 / 2}^{e}\right\}\right)+\frac{1}{\Delta x} \int_{I_{j}}\left(\frac{\rho}{\rho^{e}}-\frac{\bar{\rho}_{j}}{\bar{\rho}_{j}^{e}}\right) p_{x}^{e} \mathrm{~d} x,
$$

where $\rho_{j+1 / 2}^{e}$ is replaced by $\left\{\rho_{j+1 / 2}^{e}\right\}$. The integral of the source term in the third equation is still approximated by the Gaussian quadrature rule, and the numerical flux takes a similar form as in (2.9):

$$
\hat{f}_{j+1 / 2}=\frac{1}{2}\left[f\left(U_{j+1 / 2}^{-}\right)+f\left(U_{j+1 / 2}^{+}\right)-\alpha_{j}^{\prime}\left(\widetilde{U}_{j+1 / 2}^{+}-\widetilde{U}_{j+1 / 2}^{-}\right)\right],
$$

with the coefficient $\alpha^{\prime}$ being defined as

$$
\alpha^{\prime}=\alpha \max _{j}\left(\bar{\rho}_{j}^{e}, \bar{p}_{j}^{e}\right)
$$

and $\widetilde{U}$ is given by

$$
\widetilde{U}=\left(\frac{\rho}{\rho^{e}(x)}, \frac{\rho u}{\rho^{e}(x)}, \frac{E}{p^{e}(x)}\right)
$$

which ensures that $\widetilde{U}=$ const and the effect of the viscosity terms $\left(\widetilde{U}_{j+1 / 2}^{+}-\widetilde{U}_{j+1 / 2}^{-}\right)$disappears at the steady state.

Following the same proof, we can show that the semi-discrete WENO schemes (2.1), combined with (2.17) and (2.18), are well-balanced for the polytropic steady state solutions (1.10) of the Euler equations (2.15) under the gravitational field $\phi(x)$. 


\section{One-dimensional numerical results}

In this section, we carry out extensive one-dimensional numerical experiments to demonstrate the performances of the resulting well-balanced WENO scheme. In all the computations, we use the third order TVD Runge-Kutta time discretization (2.4) and take CFL $=0.6$.

Following [9], we define the sound crossing time $\tau_{\text {sound }}$ as

$$
\tau_{\text {sound }}=2 \int_{I} \frac{1}{c}(x) \mathrm{dx}
$$

to characterize the time scale on which a model reacts to perturbations of its equilibrium, with $c(x)=\sqrt{\gamma p / \rho}$ being the sound speed.

\subsection{Shock tube under gravitational field}

In this standard Sod test, the initial conditions are given by

$$
(\rho, u, p)= \begin{cases}(1,0,1) & \text { if } x \leq 0.5 \\ (0.125,0,0.1) & \text { otherwise }\end{cases}
$$

on a unit computational domain with a constant gravitational field $g=\phi_{x}=1$. We compute this problem up to $t=0.2$. The numerical results with 100 uniform meshes, compared with the reference solutions obtained with much refined 2000 meshes, are shown in Fig. 3.1. Due to the gravitational force acting in the left direction, the density distribution is pulled towards left, and negative velocity appears in some regions. We observe that the discontinuities in this test problem is well solved by the proposed WENO methods on the relatively coarse mesh with 100 cells, and the numerical solutions agree well with the reference ones.

\subsection{One-dimensional isothermal equilibrium in a linear gravita- tional field}

The purpose of this test case, used in [12, 22, 19], is to demonstrate the well-balanced property of our proposed methods on an isothermal equilibrium, as well as their capability 

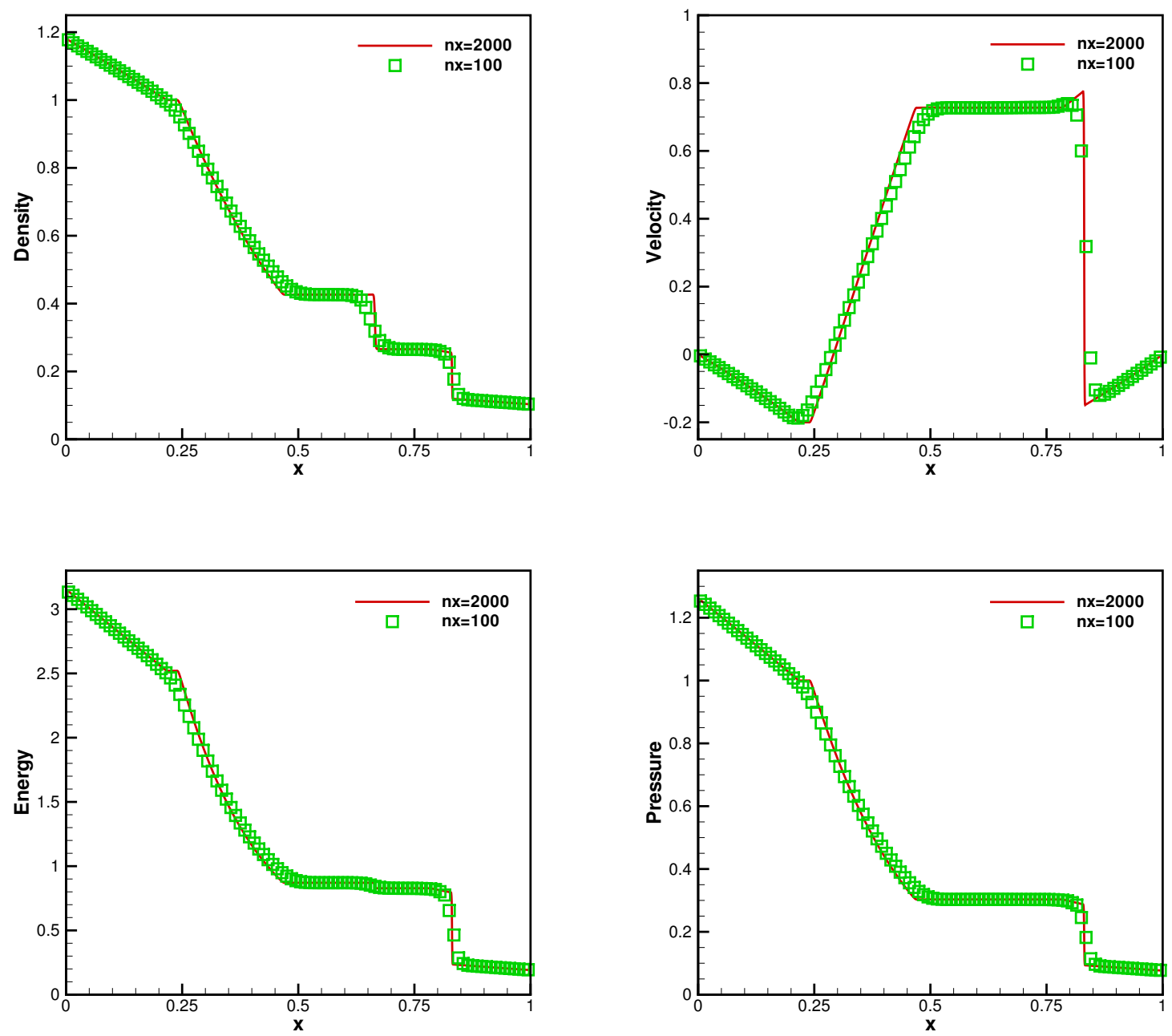

Fig. 3.1: The numerical solutions of the shock tube problem under gravitational field in Sect. 3.1 at time $t=0.2$. Top left: density distribution; Top right: velocity distribution; Bottom left: energy distribution; Bottom right: pressure distribution. 
Table 3.1: $L^{1}$ errors for different precisions for the steady state solution in Sect. 3.2.

\begin{tabular}{lllll}
\hline $\mathrm{N}$ & Precision & $\rho$ & $\rho u$ & $E$ \\
\hline \multirow{2}{*}{100} & Single & $2.63 \mathrm{E}-7$ & $3.28 \mathrm{E}-7$ & $4.40 \mathrm{E}-7$ \\
& Double & $4.31 \mathrm{E}-14$ & $6.16 \mathrm{E}-14$ & $7.84 \mathrm{E}-14$ \\
200 & Single & $3.81 \mathrm{E}-7$ & $9.63 \mathrm{E}-7$ & $5.34 \mathrm{E}-7$ \\
& Double & $1.03 \mathrm{E}-15$ & $1.30 \mathrm{E}-15$ & $1.75 \mathrm{E}-15$ \\
\hline
\end{tabular}

for capturing the small perturbation of such a steady state. We set the computational domain as $[0,1]$, and consider an ideal gas with $\gamma=1.4$ under the linear gravitational field $\phi_{x}=g=1$ acting in the negative $x$ direction. The isothermal equilibrium solution takes the form of (1.8), given by

$$
\rho_{0}(x)=p_{0}(x)=\exp (-x) \text { and } u_{0}(x)=0 .
$$

We first verify the well-balanced property of the WENO scheme. The initial condition is taken as the steady state solution (1.8), which should be exactly preserved by any wellbalanced methods. The solutions are computed until $t=2$ (the reference sound crossing time $\left.\tau_{\text {sound }} \approx 1.69\right)$ using both 100 and 200 uniform cells. In order to demonstrate that the steady state is indeed maintained up to the round-off error, we use single precision and double precision respectively to perform the computation. The $L^{1}$ errors of $\rho, \rho u$ and $E$ are shown in Table 3.1, from which we can clearly observe that the errors are all at the level of round-off error for different precisions. This verifies the expected well-balanced property.

Next, we demonstrate the advantage of well-balanced methods in capturing a small perturbation of the isothermal steady state solution (3.2). The density and velocity are kept the same, but a small perturbation is added to the initial pressure state, i.e.,

$$
p(x, 0)=p_{0}(x)+\eta \exp \left(-100(x-0.5)^{2}\right)
$$

where $\eta$ is a non-zero perturbation parameter. Two cases have been considered: $\eta=0.01$ and $\eta=0.0001$. The pressure perturbations at $t=0.25$ on a mesh with 200 cells, compared with 

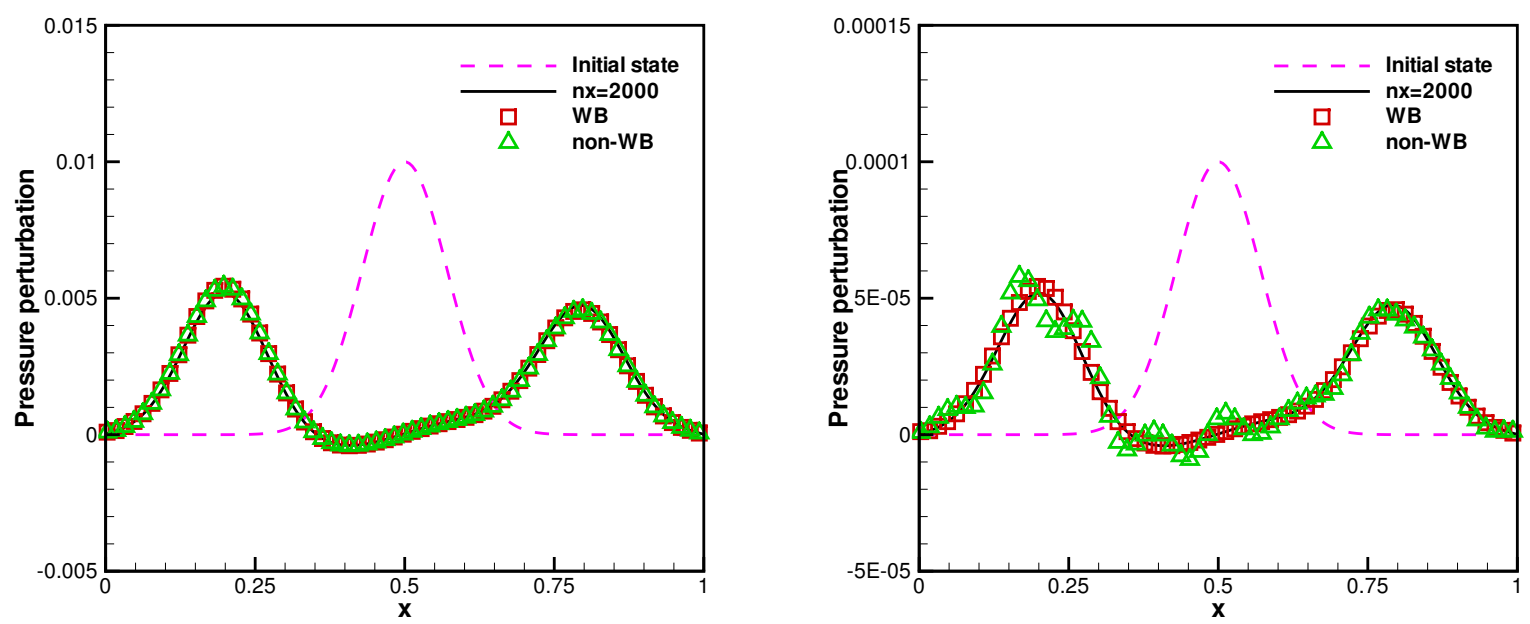

Fig. 3.2: The pressure perturbation of a hydrostatic solution in Section 3.2. The result of the well-balanced (denoted by WB) scheme with 200 and 2000 cells, and that of the nonwell-balanced (denoted by non-WB) scheme with 200 cells at time $t=0.25$. Left: $\eta=0.01$; Right: $\eta=0.0001$.

a reference solution obtained with much refined 2000 cells, are shown in Fig. 3.2. We also include the initial pressure perturbation in the plot as a dashed line. For comparison, we run the same numerical test using non-well-balanced WENO methods, with a straightforward numerical integration of the source term, and include their results in Fig. 3.2. We can easily observe that the well-balanced methods provide numerical solutions that agree well with the reference solutions for both cases. Notice that the non-well-balanced WENO scheme do not perform well for the small perturbation case on a relatively coarse mesh with 200 cells. This phenomenon demonstrates the importance of the well-balanced property in capturing small perturbations to equilibrium states.

\subsection{One-dimensional gas falling into a fixed external potential}

In this test taken from $[18,19,22]$, we verify the performance of well-balanced methods for the isothermal equilibrium in a more general gravitational field. The isothermal steady state 
Table 3.2: $L^{1}$ errors for different precisions for the steady state solution in Sect. 3.3.

\begin{tabular}{lllll}
\hline $\mathrm{N}$ & Precision & $\rho$ & $\rho u$ & $E$ \\
\hline \multirow{2}{*}{100} & Single & $3.05 \mathrm{E}-7$ & $4.14 \mathrm{E}-7$ & $3.03 \mathrm{E}-7$ \\
& Double & $2.65 \mathrm{E}-14$ & $2.41 \mathrm{E}-14$ & $7.34 \mathrm{E}-14$ \\
200 & Single & $2.74 \mathrm{E}-8$ & $1.72 \mathrm{E}-8$ & $4.14 \mathrm{E}-8$ \\
& Double & $2.71 \mathrm{E}-15$ & $7.77 \mathrm{E}-15$ & $1.11 \mathrm{E}-15$ \\
\hline
\end{tabular}

takes the form of

$$
\rho=\rho_{0} \exp \left(-\frac{\phi}{R T}\right), \quad u=0 \text { and } p=R T \rho_{0} \exp \left(-\frac{\phi}{R T}\right),
$$

with a constant temperature $T$, and the gravitational field is given by the sine wave form:

$$
\phi(x)=-\phi_{0} \frac{L}{2 \pi} \sin \frac{2 \pi x}{L},
$$

where $L$ is the computational domain length, and $\phi_{0}$ is the amplitude.

We first verify the well-balanced property of the proposed WENO schemes. We take the initial conditions as in (3.3) with parameters $\rho_{0}=1, R=1, T=0.6866, L=64, \gamma=$ $5 / 3$, and $\phi_{0}=0.02$. The solutions are computed up to $t=50$ (the reference sound crossing time $\tau_{\text {sound }} \approx 120$ ) using both 100 and 200 uniform cells. We use both single and double precisions to carry out the computation. The $L^{1}$ errors of $\rho, \rho u$ and $E$ are presented in Table 3.2 , from which we can clearly observe that the errors are all at the level of round-off error for different precisions.

Next, we impose a small perturbation to the steady state (1.7), and let the solution run for a long time until it converges to an isothermal hydrostatic state. We would like to compare the performance of well-balanced and non-well-balanced schemes for this simple test.

Taking the initial data as

$$
\rho=\rho_{0} \exp \left(-\frac{\phi}{R T}\right), \quad u=0, \quad p=R T \rho_{0} \exp \left(-\frac{\phi}{R T}\right)+0.001 \exp \left(-10(x-32)^{2}\right),
$$


with the same parameters used in the well-balanced test, we run the simulation for 1,000,000 time steps with 64 uniform cells. The stopping time $T$ is 179980.53 , which is much larger than the reference sound crossing time $\tau_{\text {sound }} \approx 120$. The numerical results at the final time are shown in Fig. 3.3. For comparison, we also run the same test with non-well-balanced WENO schemes and include their numerical results in the figures. It is easy for us to observe that the constant velocity and constant temperature distributions of the isothermal equilibrium state are correctly captured by the well-balanced WENO schemes, while the non-well-balanced schemes fail to achieve this.

\subsection{Polytropic equilibrium in a linear gravitational field}

The purpose of the test is to investigate the performance of our proposed scheme near the polytropic equilibrium states. We consider a polytropic hydrostatic atmosphere in the linear gravitational field with $\phi(x)=g x$. The polytropic steady state solutions, taken from [9], are given by

$$
\begin{aligned}
& \rho(x)=\left(\rho_{0}^{\gamma-1}-\frac{1}{K_{0}} \frac{\gamma-1}{\gamma} g x\right)^{\frac{1}{\gamma-1}} \\
& u(x)=0 \\
& p(x)=K_{0} \rho(x)^{\gamma}
\end{aligned}
$$

with $g=1, \gamma=5 / 3, \rho_{0}=1, p_{0}=1$ and $K_{0}=p_{0} / \rho_{0}^{\gamma}$ on a computational domain $[0,2]$.

We first show an example to demonstrate the well-balanced property of the proposed WENO schemes. The initial condition (3.4) should be exactly preserved by the well-balanced scheme. In order to demonstrate that the steady state is indeed maintained up to the round-off error, we use single precision and double precision respectively to perform the computation. We compute the solution until $t=4$ (the sound crossing time $\tau_{\text {sound }} \approx 3.92$ ) using both 100 and 200 uniform mesh cells, and present the $L^{1}$ errors of numerical solutions in Table 3.3. We can clearly observe that the numerical errors are all at the level of round-off error for different precisions, which verifies the desired well-balanced property.

Next, we impose a small perturbation to the polytropic hydrostatic equilibrium, and 

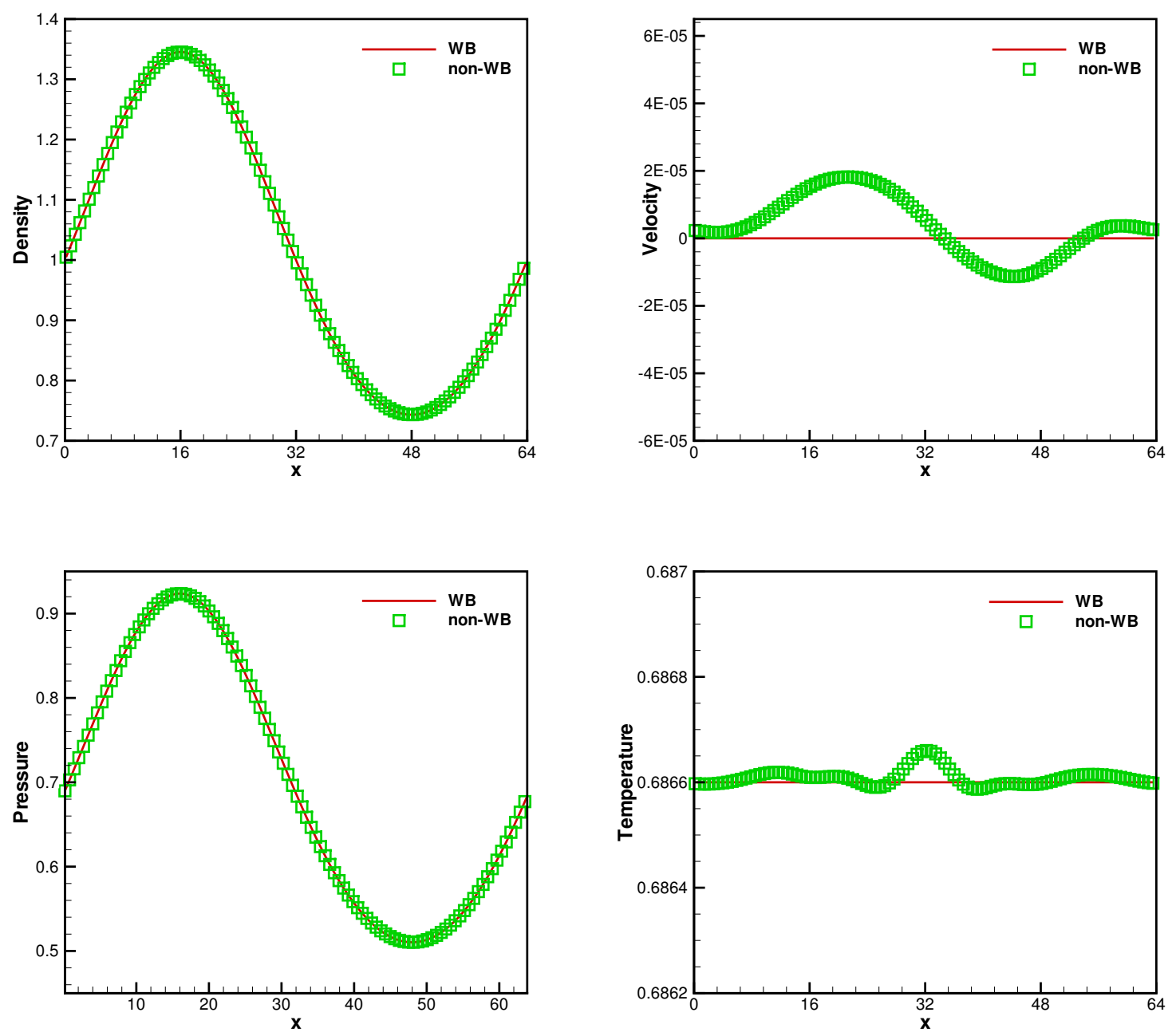

Fig. 3.3: The numerical solutions of well-balanced scheme (solid line, denoted by WB) and non-well-balanced scheme (square box, denoted by non-WB) for the convergence test in Section 3.3 after 1,000,000 time steps. Top left: density distribution; Top right: velocity distribution; Bottom left: pressure distribution; Bottom right: temperature distribution. 
Table 3.3: $L^{1}$ errors for different precisions for the steady state solution in Section 3.4.

\begin{tabular}{lllll}
\hline $\mathrm{N}$ & Precision & $\rho$ & $\rho u$ & $\mathrm{E}$ \\
\hline \multirow{2}{*}{100} & Single & $1.01 \mathrm{E}-6$ & $1.48 \mathrm{E}-7$ & $8.27 \mathrm{E}-7$ \\
& Double & $1.33 \mathrm{E}-15$ & $1.55 \mathrm{E}-16$ & $8.75 \mathrm{E}-16$ \\
200 & Single & $4.53 \mathrm{E}-6$ & $5.24 \mathrm{E}-7$ & $2.83 \mathrm{E}-7$ \\
& Double & $3.34 \mathrm{E}-15$ & $5.10 \mathrm{E}-15$ & $2.67 \mathrm{E}-16$ \\
\hline
\end{tabular}

compare the performance of well-balanced and non-well-balanced schemes. At the bottom of the atmosphere, we add a periodic velocity perturbation of the form

$$
u(0, t)=A \sin (4 \pi t)
$$

with $A=10^{-6}$ as a small perturbation. The solutions are computed at the time $t=1.5$, before these perturbation waves propagate to the upper boundary $x=2$. As the wave moves through the domain, its amplitude is modified by the density and pressure stratification because of the gravity.

In Fig. 3.4, we present the pressure perturbations and velocity of the solutions at time $t=1.5$ on a coarse mesh with 200 cells. For comparison, we include the reference solutions obtained with much refined 2000 cells. In addition, we run the same numerical test using non-well-balanced WENO schemes on 200 cells, and show their results in the figure. It is obvious that the results of well-balanced WENO schemes are in good agreement with the reference solutions, while the perturbations of non-well-balanced schemes are not consistent with the reference solutions, especially for the region when $x>1.5$.

Lastly, we keep the setting in the previous test, but impose a large amplitude perturbation instead. The similar test case as above, but with $A=0.1$, is tested. We again evolve the simulation until $t=1.5$. The numerical results by both well-balanced and non-well-balanced WENO schemes with 200 cells are displayed in Fig. 3.5, compared against the reference solutions (with 2000 cells). From the figures, we can observe that both methods are able to capture the large perturbation waves well. This agrees with our expectation, as we hope the 

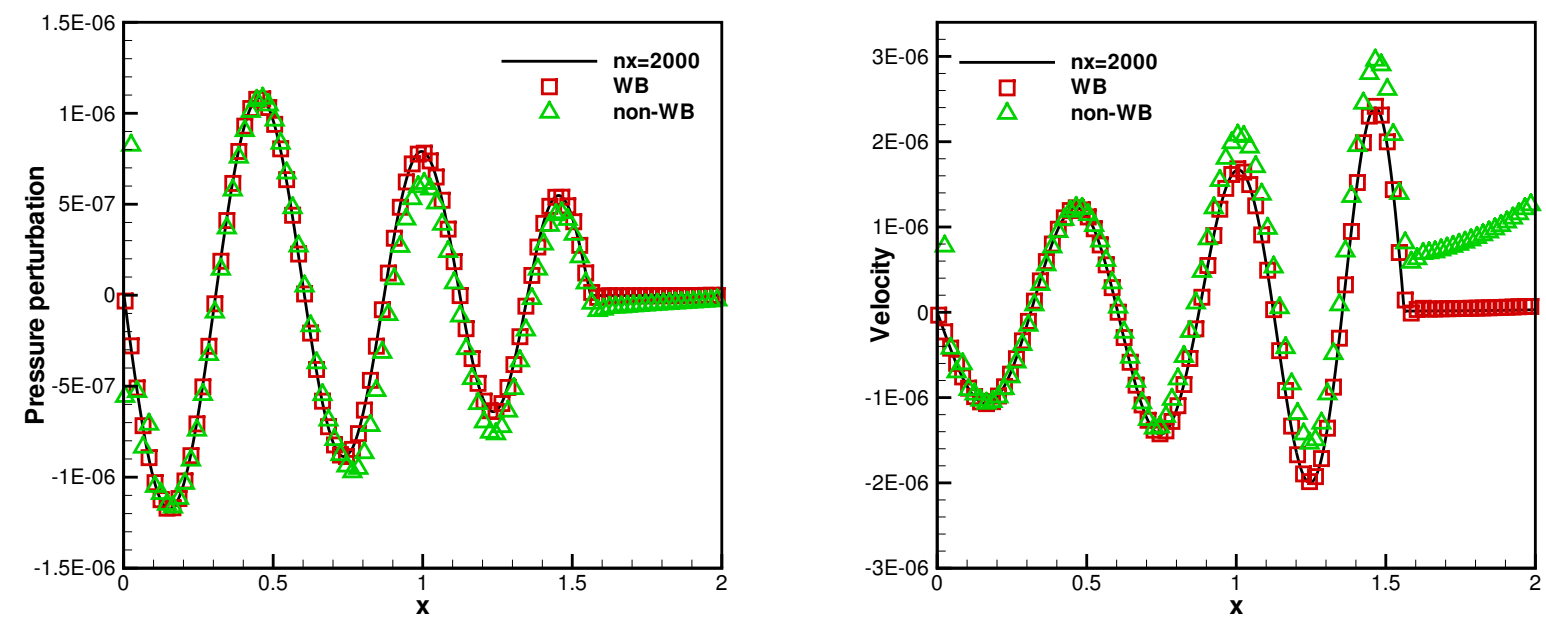

Fig. 3.4: Small amplitude waves traveling up the isentropic hydrostatic atmosphere. The pressure perturbations and velocity of a hydrostatic solution in Section 3.4. The result of the well-balanced (denoted by WB) scheme with 200 and 2000 cells, and that of the nonwell-balanced (denoted by non-WB) scheme with 200 cells at $t=1.5$.

well-balanced methods to perform similarly as non-well-balanced methods when far away from the steady states.

\section{Extension to multi-dimensional case}

The one-dimensional well-balanced techniques for both the isothermal and polytropic equilibrium solutions can be extended to multi-dimension. In this section, we present well-balanced finite volume WENO schemes to multi-dimensional Euler equations (1.1) under the gravitational field $\phi$ on structured meshes. The multi-dimensional isothermal steady state solution we are interested to preserve is given by

$$
\rho=\rho_{0} \exp \left(-\frac{\phi}{R T_{0}}\right), \quad \mathbf{u}=0, \quad p=R T \rho=R T \rho_{0} \exp \left(-\frac{\phi}{R T_{0}}\right),
$$

and the polytropic equilibrium takes the form of

$$
\rho=\left(\frac{\nu-1}{K \nu}(C-\phi)\right)^{\frac{1}{\nu-1}}, \quad \mathbf{u}=0, \quad p=\frac{1}{K^{\frac{1}{\nu-1}}}\left(\frac{\nu-1}{\nu}(C-\phi)\right)^{\frac{\nu}{\nu-1}} .
$$



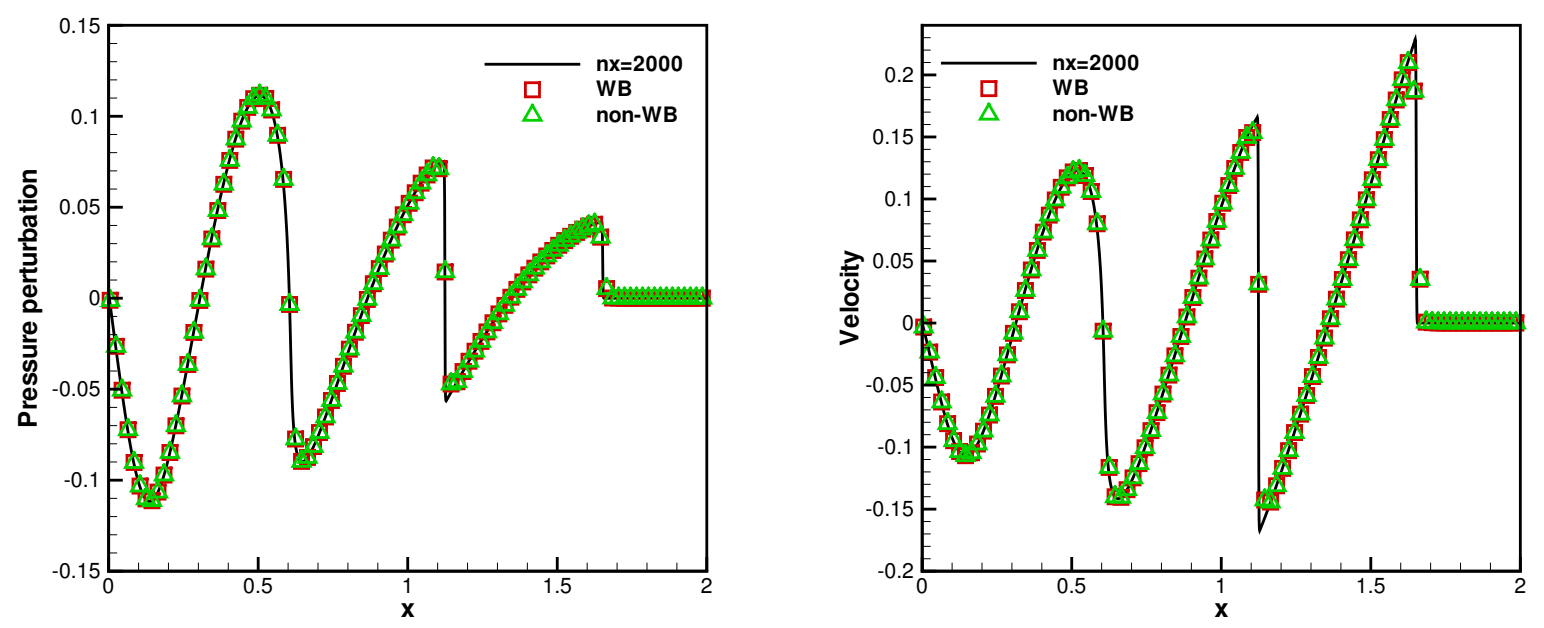

Fig. 3.5: Large amplitude waves traveling up the isentropic hydrostatic atmosphere. The pressure perturbations and velocity of a hydrostatic solution in Section 3.4. The result of the well-balanced (denoted by WB) scheme with 200 and 2000 cells, and that of the nonwell-balanced (denoted by non-WB) scheme with 200 cells at $t=1.5$.

For ease of presentation, we restricted our discussion in this section to two spatial dimensions only, although the algorithm can be easily designed for three spatial dimensions as well.

We discretize the computational domain into cells $I_{i, j}=\left[x_{i-\frac{1}{2}}, x_{i+\frac{1}{2}}\right] \times\left[y_{j-\frac{1}{2}}, y_{j+\frac{1}{2}}\right]$. For simplicity, we assume a uniform mesh is used. The sizes of each rectangle cell are denoted by $\Delta x$ and $\Delta y$, with $\lambda_{1}=\Delta t / \Delta x, \lambda_{2}=\Delta t / \Delta y$. The integrals will be approximated by quadratures with sufficient accuracy. Let us assume that we use a Gaussian quadrature with $L$ points. We assume

$$
S_{i}^{x}=\left\{x_{i}^{\beta}: \beta=1, \cdots, L\right\}, \quad S_{j}^{y}=\left\{y_{j}^{\beta}: \beta=1, \cdots, L\right\}
$$

denote the Gaussian quadrature points on $\left[x_{i-\frac{1}{2}}, x_{i+\frac{1}{2}}\right]$ and $\left[y_{j-\frac{1}{2}}, y_{j+\frac{1}{2}}\right]$, respectively. For instance, $\left(x_{i-\frac{1}{2}}, y_{j}^{\beta}\right)(\beta=1, \cdots, L)$ are the Gaussian quadrature points on the left edge of the $(i, j)$ cell. Let $w_{\beta}$ be the Gaussian quadrature weights for the interval $\left[-\frac{1}{2}, \frac{1}{2}\right]$. The 
two-dimensional finite volume WENO scheme is then given by

$$
\frac{d}{d t} \bar{U}_{i j}(t)=-\frac{1}{\Delta x}\left(\widehat{f}_{i+\frac{1}{2}, j}-\widehat{f}_{i-\frac{1}{2}, j}\right)-\frac{1}{\Delta y}\left(\widehat{g}_{i, j+\frac{1}{2}}-\widehat{g}_{i, j-\frac{1}{2}}\right)+\frac{1}{\Delta x \Delta y} \int_{I_{i, j}} S(h, \phi) \mathrm{d} x \mathrm{~d} y
$$

where

$$
\widehat{f}_{i+\frac{1}{2}, j}=\sum_{\beta} w_{\beta} F\left(U_{x_{i+\frac{1}{2}}, y_{j}^{\beta}}^{-} U_{x_{i+\frac{1}{2}}, y_{j}^{\beta}}^{+}\right),
$$

is an approximation to the integration of $f$ in $y$-direction:

$$
\frac{1}{\Delta y} \int_{y_{j-\frac{1}{2}}}^{y_{j+\frac{1}{2}}} f\left(U\left(x_{i+\frac{1}{2}}, y, t\right)\right) \mathrm{d} y
$$

where the Lax-Friedrichs flux

$$
F\left(a_{1}, a_{2}\right)=\frac{1}{2}\left(f\left(a_{1}\right)+f\left(a_{2}\right)-\alpha_{1}\left(a_{2}-a_{1}\right)\right), \quad \alpha_{1}=\max (|u|+\sqrt{\gamma p / \rho}),
$$

is used. $U_{x_{i+\frac{1}{2}}, y_{j}^{\beta}}^{ \pm}$are the high order pointwise approximations to $U\left(\left(x_{i+\frac{1}{2}}, y_{j}^{\beta}, t\right)\right.$ by a WENO reconstruction procedure. For rectangular meshes, a practical way to perform the reconstruction in two space dimensions is given as follows. We first perform a one-dimensional reconstruction in one of the directions (e.g., the y-direction), obtaining one dimensional cell averages of the function $\mathrm{u}$ in the other direction (e.g., the x-direction). A reconstruction in the other direction is then applied to obtain the approximated point values. The other flux $\widehat{g}_{i, j+\frac{1}{2}}$ is computed in the similar way with

$$
G\left(a_{1}, a_{2}\right)=\frac{1}{2}\left(g\left(a_{1}\right)+g\left(a_{2}\right)-\alpha_{2}\left(a_{2}-a_{1}\right)\right), \quad \alpha_{2}=\max (|v|+\sqrt{\gamma p / \rho}) .
$$

To extend our well-balanced finite volume WENO scheme in Section 2 to two dimensions, we first rewrite the source term using the targeting equilibrium states, as in (2.5) for the isothermal solution or (2.15) for the polytropic case. Below, we take the polytropic case as an example to demonstrate the two-dimensional well-balanced WENO schemes. The Euler 
equations (1.1) can be reformulated as

$$
\begin{aligned}
& \rho_{t}+(\rho u)_{x}+(\rho v)_{y}=0, \\
& (\rho u)_{t}+\left(\rho u^{2}+p\right)_{x}+(\rho u v)_{y}=\frac{\rho}{\rho^{e}} p_{x}^{e}, \\
& (\rho v)_{t}+(\rho u v)_{x}+\left(\rho v^{2}+p\right)_{y}=\frac{\rho}{\rho^{e}} p_{y}^{e}, \\
& E_{t}+((E+p) u)_{x}+((E+p) v)_{y}=-\rho u \phi_{x}-\rho v \phi_{y},
\end{aligned}
$$

with $\rho^{e}(x)$ and $p^{e}(x)$ defined in (2.14).

Following the same technique as stated in Section 2, we decompose the integral of the source term in the second equation as

$$
\begin{aligned}
& \frac{1}{\Delta x \Delta y} \int_{I_{i j}} S^{[2]} \mathrm{d} x \mathrm{~d} y=\frac{1}{\Delta x \Delta y} \int_{I_{i j}} \frac{\rho}{\rho^{e}} p_{x}^{e} \mathrm{~d} x \mathrm{~d} y \\
& \quad=\frac{1}{\Delta x \Delta y} \frac{\bar{\rho}_{i j}}{\bar{\rho}_{i j}^{e}}\left(\int_{y_{j-\frac{1}{2}}}^{y_{j+\frac{1}{2}}} p_{x_{i+\frac{1}{2}}, y}^{e} \mathrm{~d} y-\int_{y_{j-\frac{1}{2}}}^{y_{j+\frac{1}{2}}} p_{x_{i-\frac{1}{2}}, y}^{e} \mathrm{~d} y\right)+\frac{1}{\Delta x \Delta y} \int_{I_{i j}}\left(\frac{\rho}{\rho^{e}}-\frac{\bar{\rho}_{i j}}{\bar{\rho}_{i j}^{e}}\right) p_{x}^{e} \mathrm{~d} x \mathrm{~d} y,
\end{aligned}
$$

and approximate it by

$$
\begin{aligned}
& \frac{1}{\Delta x \Delta y} \int_{I_{i j}} S^{[2]} \mathrm{d} x \mathrm{~d} y \approx \frac{1}{\Delta x \Delta y} \frac{\bar{\rho}_{i j}}{\bar{\rho}_{i j}^{e}}\left(\sum_{\beta} w_{\beta}\left\{p_{x_{i+\frac{1}{2}}, y_{j}^{\beta}}^{e}\right\}-\sum_{\beta} w_{\beta}\left\{p_{x_{i-\frac{1}{2}}, y_{j}^{\beta}}^{e}\right\}\right) \\
& +\frac{1}{\Delta x \Delta y} \int_{I_{i j}}\left(\frac{\rho}{\rho^{e}}-\frac{\bar{\rho}_{i j}}{\bar{\rho}_{i j}^{e}}\right) p_{x}^{e} \mathrm{~d} x \mathrm{~d} y .
\end{aligned}
$$

The approximation to the integral of the source term in the third equation follows the same idea. The numerical flux takes a similar form as in (2.9)

$$
\widehat{f}_{j+1 / 2}=\frac{1}{2}\left[f\left(U_{j+1 / 2}^{-}\right)+f\left(U_{j+1 / 2}^{+}\right)-\alpha_{j}^{\prime}\left(\widetilde{U}_{j+1 / 2}^{+}-\widetilde{U}_{j+1 / 2}^{-}\right)\right] .
$$

where $\widetilde{U}$ is given by

$$
\widetilde{U}=\left(\frac{\rho}{\rho^{e}(x)}, \frac{\rho u}{\rho^{e}(x)}, \frac{\rho v}{\rho^{e}(x)}, \frac{E}{p^{e}(x)}\right),
$$

which ensures that $\widetilde{U}=$ const and the effect of the viscosity terms $\left(\widetilde{U}_{j+1 / 2}^{+}-\widetilde{U}_{j+1 / 2}^{-}\right)$disappears at the steady state. All the desired properties proved in the one-dimensional case, such as high order accuracy and the well-balanced property, are still valid in the two-dimensional case. 


\section{Two-dimensional numerical results}

In this section, we carry out some two-dimensional numerical experiments to demonstrate the performances of the resulting well-balanced WENO scheme. The third order TVD RungeKutta time discretization (2.4) and $\mathrm{CFL}=0.6$ are also used in all the computations below.

\subsection{Testing the orders of accuracy}

In this example, we test the numerical orders of accuracy based on the two-dimensional equations (1.1) with a linear gravitational field with $\phi_{x}=\phi_{y}=1$ as in [22]. We consider the exact solution taking the form of

$$
\begin{aligned}
& \rho(x, y, t)=1+0.2 \sin \left(\pi\left(x+y-t\left(u_{0}+v_{0}\right)\right)\right) \\
& u(x, y, t)=u_{0} \\
& v(x, y, t)=v_{0} \\
& p(x, y, t)=p_{0}+t\left(u_{0}+v_{0}\right)-x-y+0.2 \cos \left(\pi\left(x+y-t\left(u_{0}+v_{0}\right)\right)\right) / \pi
\end{aligned}
$$

on a square domain $[0,2] \times[0,2]$. We take $u_{0}=v_{0}=1$ and $p_{0}=4.5$ in this test case, and apply the exact solutions at the boundaries. The time step $\Delta t$ is taken to be proportional to $(1 / \Delta x+1 / \Delta y)^{-5 / 3}$. We run the simulation up to $t=0.1$ and compute the numerical errors by comparing with the exact solutions. The $L^{1}$ errors and orders of accuracy are shown in Table 5.1, where we can clearly see that the expected high order accuracy is achieved for the proposed well-balanced WENO scheme.

\subsection{Two-dimensional isothermal equilibrium solution}

The purpose of this test case is to demonstrate the well-balanced property of our proposed methods on an isothermal equilibrium solution and their capacity for capturing the propagation of small wave perturbation in the two-dimensional case. Consider an ideal gas with $\gamma=1.4$ and the linear gravitational field $\phi_{x}=\phi_{y}=g$. As in [22], we consider the isothermal 
Table 5.1: $L^{1}$ errors and numerical orders of accuracy for the example in Sect. 5.1.

\begin{tabular}{lcccccccc}
\hline \multirow{2}{*}{ Cells } & $\rho$ & \multicolumn{2}{c}{$\rho u$} & \multicolumn{2}{c}{$E$} \\
\cline { 2 - 8 } & $L^{1}$ error & Order & $L^{1}$ error & Order & $L^{1}$ error & Order & $L^{1}$ error & Order \\
\hline $8 \times 8$ & $1.75 \mathrm{E}-02$ & & $1.97 \mathrm{E}-02$ & & $1.97 \mathrm{E}-02$ & & $1.13 \mathrm{E}-01$ & \\
\hline $16 \times 16$ & $2.21 \mathrm{E}-03$ & 2.98 & $2.83 \mathrm{E}-03$ & 2.80 & $2.83 \mathrm{E}-03$ & 2.80 & $1.97 \mathrm{E}-02$ & 2.52 \\
\hline $32 \times 32$ & $3.32 \mathrm{E}-04$ & 2.74 & $2.01 \mathrm{E}-04$ & 3.81 & $2.01 \mathrm{E}-04$ & 3.81 & $2.81 \mathrm{E}-03$ & 2.81 \\
\hline $64 \times 64$ & $2.34 \mathrm{E}-05$ & 3.83 & $8.14 \mathrm{E}-06$ & 4.63 & $8.14 \mathrm{E}-06$ & 4.63 & $2.02 \mathrm{E}-04$ & 3.80 \\
\hline $128 \times 128$ & $9.44 \mathrm{E}-07$ & 4.63 & $2.73 \mathrm{E}-07$ & 4.90 & $2.73 \mathrm{E}-07$ & 4.90 & $8.19 \mathrm{E}-06$ & 4.62 \\
\hline $256 \times 256$ & $2.99 \mathrm{E}-08$ & 4.98 & $3.08 \mathrm{E}-09$ & 5.02 & $3.08 \mathrm{E}-09$ & 5.02 & $2.54 \mathrm{E}-07$ & 5.01 \\
\hline
\end{tabular}

Table 5.2: $L^{1}$ errors for different precisions for the steady state solution (5.1) in Sect. 5.2.

\begin{tabular}{lllll}
\hline Precision & $\rho$ & $\rho u$ & $\rho v$ & $E$ \\
\hline Single & $5.91 \mathrm{E}-8$ & $8.94 \mathrm{E}-8$ & $8.93 \mathrm{E}-8$ & $1.28 \mathrm{E}-8$ \\
Double & $6.04 \mathrm{E}-14$ & $8.92 \mathrm{E}-14$ & $8.92 \mathrm{E}-14$ & $1.27 \mathrm{E}-14$ \\
\hline
\end{tabular}

equilibrium state

$$
\begin{aligned}
& \rho(x)=\rho_{0} \exp \left(-\frac{\rho_{0} g}{p_{0}}(x+y)\right), \\
& u(x, y)=v(x, y)=0, \\
& p(x, y)=p_{0} \exp \left(-\frac{\rho_{0} g}{p_{0}}(x+y)\right),
\end{aligned}
$$

with $\rho_{0}=1.21, p_{0}=1$ and $g=1$ on a unit square domain.

Using this equilibrium state as the initial data, we first verify their well-balanced property. We compute the solution up to $t=1$ on a mesh with $50 \times 50$ uniform cells. The $L^{1}$ errors of $\rho, \rho u, \rho v$ and $E$, using both single precision and double precision, are shown in Table 5.2, where we can easily observe that the errors are all at the level of round-off errors and the well-balanced property is obtained.

Next, we impose a small perturbation to the pressure state of the isothermal equilibrium solution (5.1):

$$
p(x, y)=p_{0} \exp \left(-\frac{\rho_{0} g}{p_{0}}(x+y)\right)+0.001 \exp \left(-\frac{\rho_{0} g}{p_{0}}\left((x-0.3)^{2}+(y-0.3)^{2}\right)\right),
$$



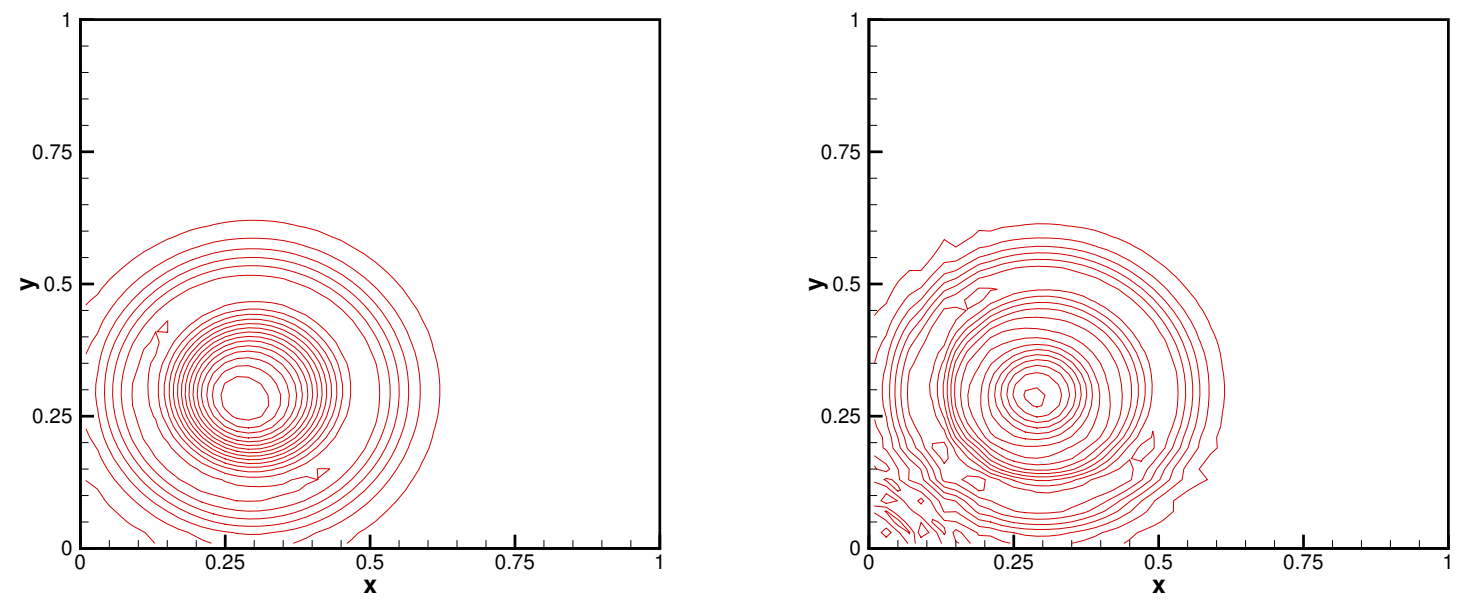

Fig. 5.1: The contours of the pressure perturbation of a two-dimensional hydrostatic solution in Section 5.2 at time $t=0.15$ with $50 \times 50$ cells. 20 uniformly spaced contour lines from 0.0003 to 0.0003 . Left: results based on well-balanced scheme. Right: results based on non-well-balanced scheme.

and keep the density and velocities to be the same. Both well-balanced WENO schemes and non-well-balanced schemes (with a straightforward integration of the source term) are simulated. The contour plots of the pressure perturbation, for the numerical solutions at $t=0.15$ with $50 \times 50$ cells and simple transmissive boundary conditions, are shown in Fig. 5.1. Fig. 5.2 contains the 3D figures of the pressure perturbations. The density perturbations are shown in Fig. 5.3. From these figures, we can observe that non-well-balanced WENO schemes are not able to capture these small perturbations on the coarse mesh, while the well-balanced ones can resolve them very well. We also refine the mesh to $200 \times 200$ uniform cells, and show their pressure perturbation results in Fig. 5.4. As we expected, the results of nonwell-balanced WENO schemes are improving on this refined mesh. There are still some small oscillations in the non-well-balanced solution, which will disappear in much refined 400 meshes. 

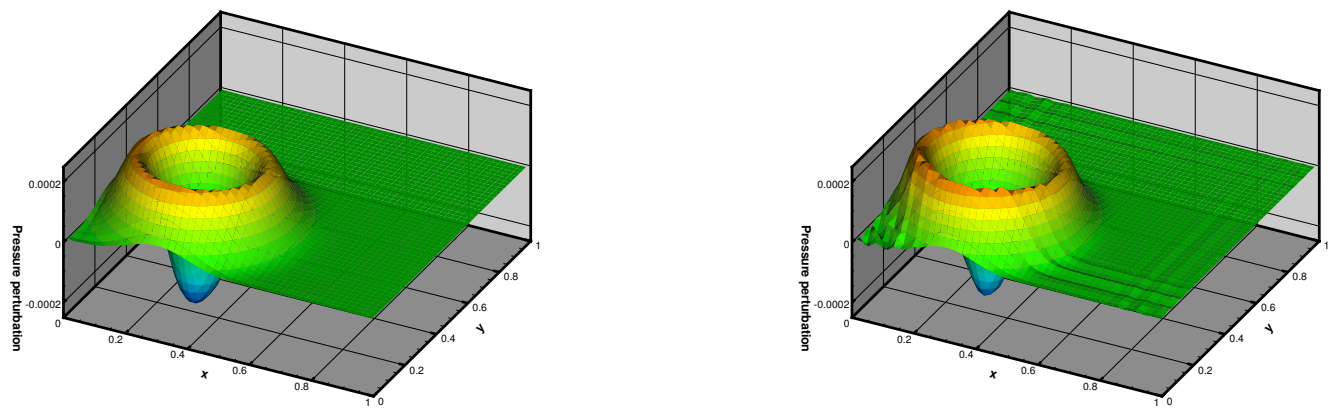

Fig. 5.2: The 3D figure of the pressure perturbation of a two-dimensional hydrostatic solution in Section 5.2 at time $t=0.15$ with $50 \times 50$ cells. Left: results based on well-balanced scheme. Right: results based on non-well-balanced scheme.
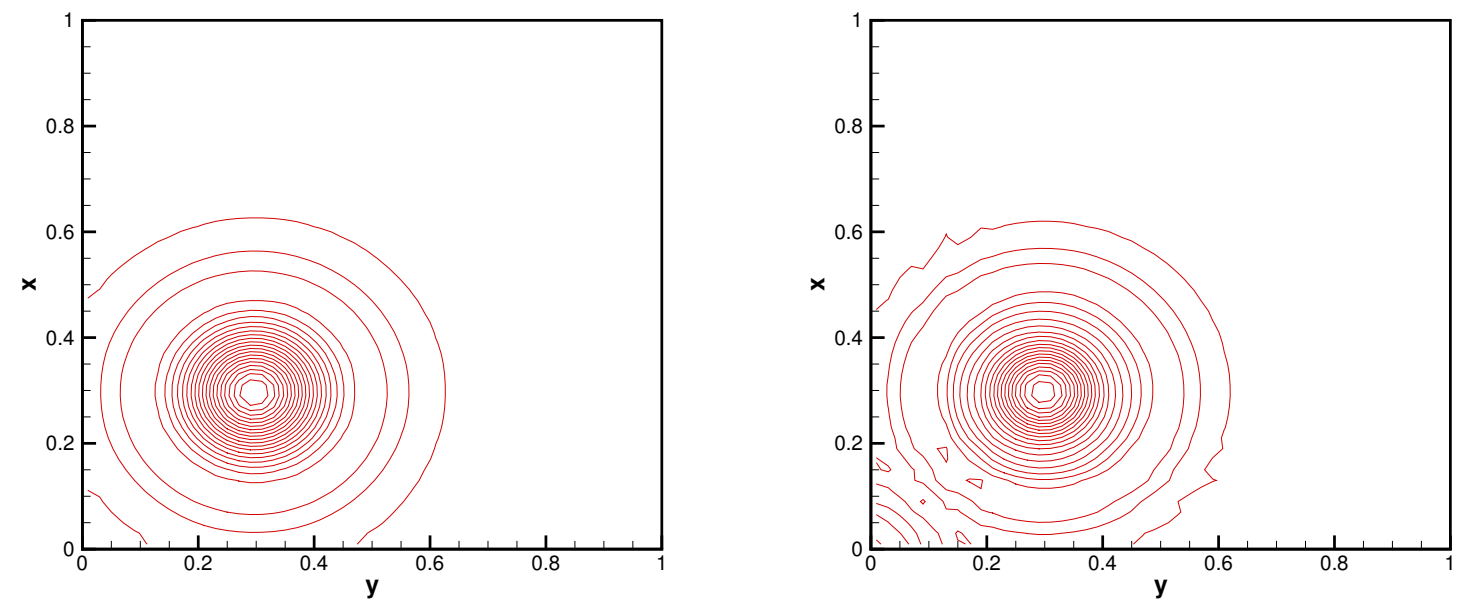

Fig. 5.3: The contours of the density perturbation of a two-dimensional hydrostatic solution in Section 5.2 at time $t=0.15$ with $50 \times 50$ cells. 20 uniformly spaced contour lines from -0.001 to 0.0002 . Left: results based on well-balanced scheme. Right: results based on non-well-balanced scheme. 

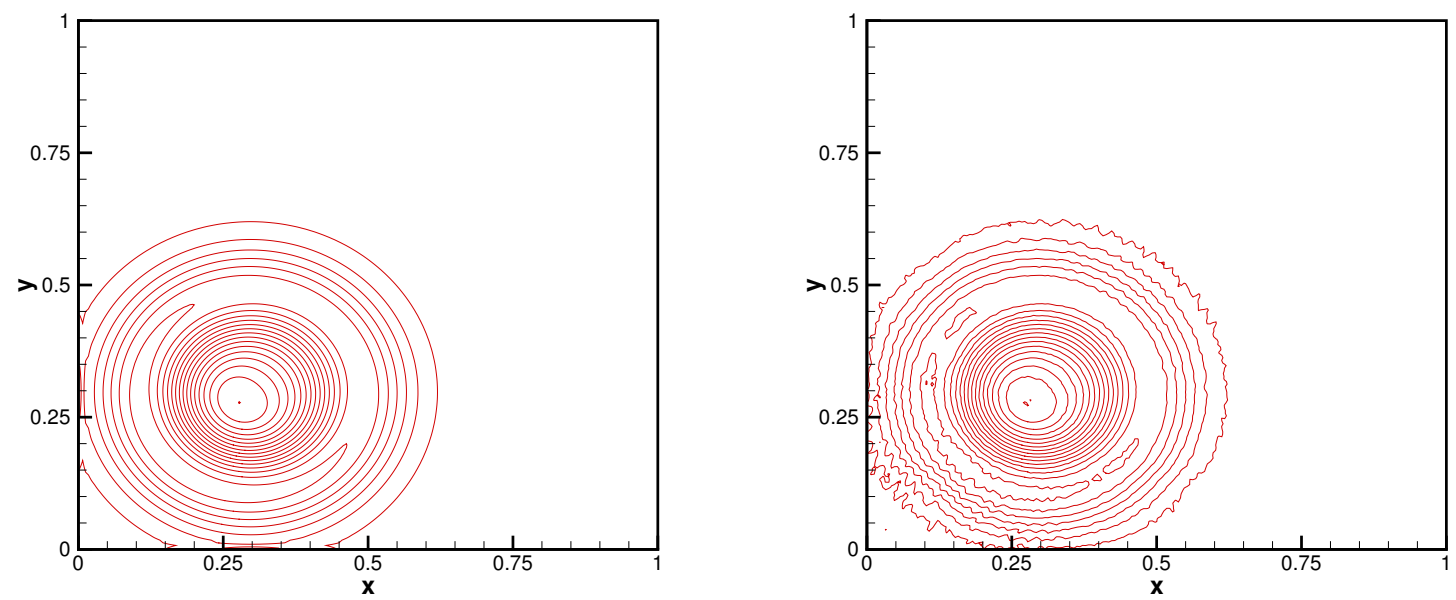

Fig. 5.4: The contours of the pressure perturbation of a two-dimensional hydrostatic solution in Section 5.2 at time $t=0.15$ with $200 \times 200$ cells. 20 uniformly spaced contour lines from -0.0003 to 0.0003 . Left: results based on well-balanced scheme. Right: results based on non-well-balanced scheme.

\subsection{Two-dimensional polytropic equilibrium solution}

In this last example, considered in [9], we verify the performance of our proposed WENO methods for a two-dimensional polytropic test case. The test is based on a static adiabatic gaseous sphere, called polytrope, which is held together by self-gravitation. From the hydrostatic equilibrium

$$
\frac{\mathrm{d} p}{\mathrm{~d} r}=-\rho \frac{\mathrm{d} \phi}{\mathrm{d} r}
$$

and Poisson's equation

$$
\frac{1}{r^{2}} \frac{\mathrm{d}}{\mathrm{d} r}\left(r^{2} \frac{\mathrm{d} \phi}{\mathrm{d} r}\right)=4 \pi g \rho
$$

in spherical symmetry with $r=\sqrt{x^{2}+y^{2}}$, one can derive the Lane-Emden equation

$$
\frac{1}{r^{2}} \frac{\mathrm{d}}{\mathrm{d} r}\left(r^{2} \gamma K \frac{\mathrm{d} \rho}{\mathrm{d} r}\right)=-4 \pi g \rho,
$$

where the polytropic relation $p=K \rho^{\gamma}$ with $K$ being constant is used. The Lane-Emden equation can be solved analytically, for three special ratios of specific heats with $\gamma=6 / 5,2, \infty$. 
Table 5.3: $L^{1}$ errors for different precisions for the steady state solution (5.5) and (5.6) in Section 5.3.

\begin{tabular}{llllll}
\hline $\mathrm{N}$ & Precision & $\rho$ & $\rho u$ & $\rho v$ & $E$ \\
\hline $50 \times 50$ & Single & $4.96 \mathrm{E}-8$ & $5.96 \mathrm{E}-8$ & $5.87 \mathrm{E}-8$ & $7.17 \mathrm{E}-8$ \\
& Double & $9.14 \mathrm{E}-16$ & $3.53 \mathrm{E}-16$ & $3.53 \mathrm{E}-16$ & $1.31 \mathrm{E}-15$ \\
\multirow{2}{*}{$100 \times 100$} & Single & $5.99 \mathrm{E}-8$ & $8.36 \mathrm{E}-8$ & $8.33 \mathrm{E}-8$ & $7.14 \mathrm{E}-8$ \\
& Double & $2.51 \mathrm{E}-15$ & $1.16 \mathrm{E}-15$ & $1.16 \mathrm{E}-15$ & $3.94 \mathrm{E}-15$ \\
\hline
\end{tabular}

Here, we take $\gamma=2$, and the analytical density and pressure are then given by

$$
\rho(r)=\rho_{c} \frac{\sin (\alpha r)}{\alpha r}, \quad p(r)=K \rho(r)^{2},
$$

with $\alpha=\sqrt{\frac{4 \pi g}{2 K}}, \rho_{c}$ being the central density of the polytrope, and the gravitational potential taking the form of

$$
\phi(r)=-2 K \rho_{c} \frac{\sin (\alpha r)}{\alpha r} .
$$

The parameters $K=g=\rho_{c}=1$ are used in the computation. The computational domain is set as $[-0.5,0.5] \times[-0.5,0.5]$. We take the exact solutions $(5.5)-(5.6)$ as the initial conditions, and set velocity to be zero.

Easy to verify that the initial condition is a steady state solution and satisfies the polytropic equilibrium. We first test the well-balanced property of our proposed WENO methods. We compute the solution up to $t=14.8\left(\approx 20 \tau_{\text {sound }}\right)$ on meshes with $50 \times 50$ and $100 \times 100$ uniform cells. In order to demonstrate that the steady state is indeed maintained up to round-off error, we use single precision and double precision to carry out the computation. The $L^{1}$ errors of $\rho, \rho u, \rho v$ and $E$ are shown in Table 5.3, where the well-balanced property can be easily observed.

Next, we impose a small perturbation to the hydrostatic equilibrium and test the capability of our methods in capturing the propagation of such small perturbations. A small Gaussian hump perturbation is added to the pressure profile (5.5), which leads to

$$
p(r)=K \rho(r)^{2}+A \exp \left(-100 r^{2}\right) .
$$



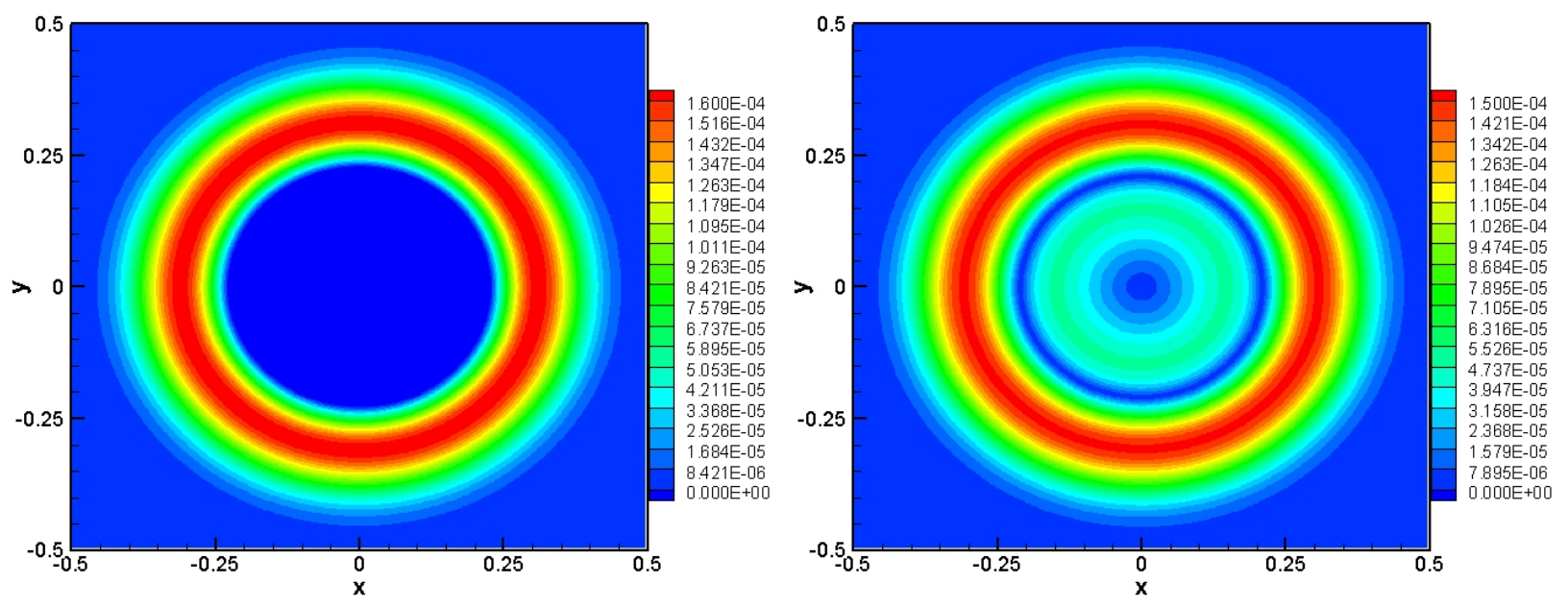

Fig. 5.5: The contours of the pressure perturbation and the velocity $\left(\sqrt{u^{2}+v^{2}}\right)$ of the small amplitude wave propagation problem in Section 5.3 by well-balanced WENO scheme at time $t=0.2$ with $200 \times 200$ cells. Left: pressure perturbation. Right: velocity.

We keep the velocity and pressure to be same, and set the amplitude parameter $A$ as $10^{-3}$.

The simulation is run up to $t=0.2$ on a mesh with $200 \times 200$ cells. The contour plots of their pressure perturbation and velocity $\sqrt{u^{2}+v^{2}}$ are shown in Fig. 5.5. The results are comparable with those in [9]. For comparison, we also include the numerical results of non-well-balanced WENO methods in Fig. 5.6. Moreover, we also show the 3D views of the pressure perturbation and the velocity $\left(\sqrt{u^{2}+v^{2}}\right)$ of the well-balanced and non-wellbalanced schemes in Fig. 5.7 and Fig. 5.8, respectively. From these figures, we can clearly observe that non-well-balanced schemes are not capable of capturing such small perturbation on the relatively coarse mesh, while the well-balanced ones can resolve it very well.

\section{Concluding remarks}

In this paper, we develop high order well-balanced finite volume WENO scheme for the Euler equations under gravitational field. This system admits the hydrostatic balance where the flux produced by the pressure is exactly canceled by the gravitational source term. 

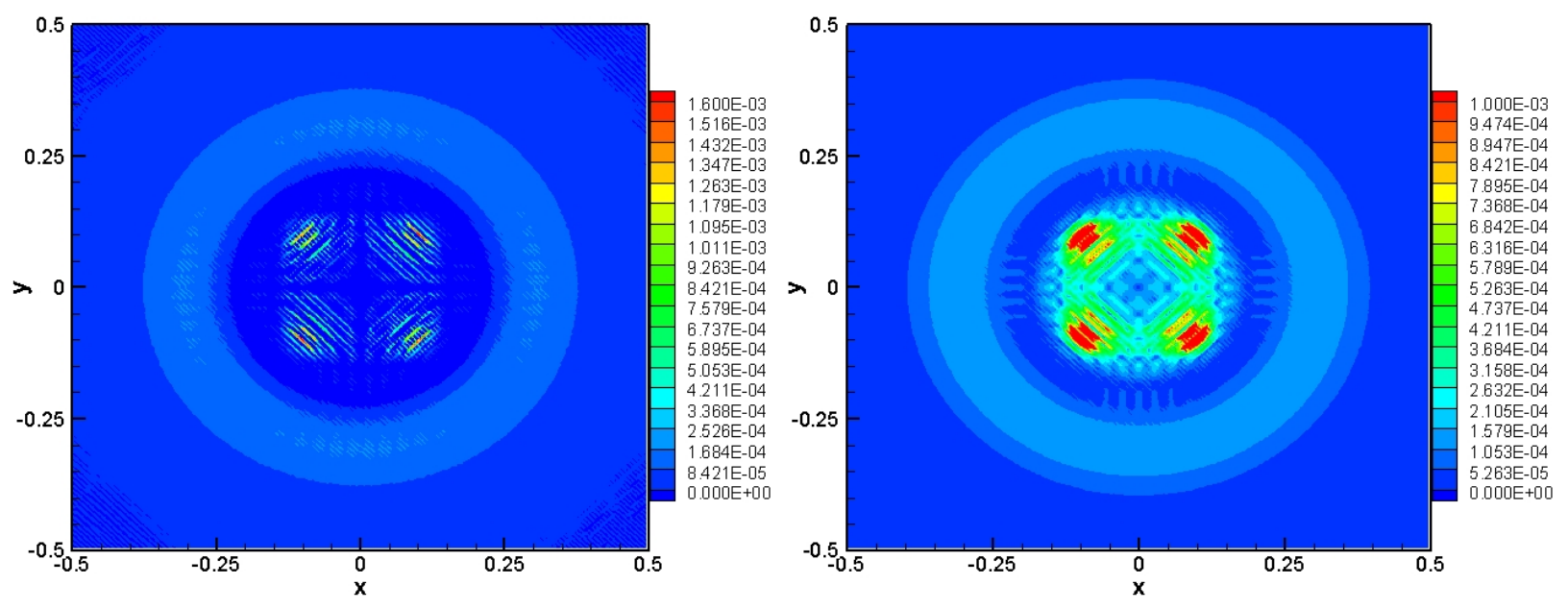

Fig. 5.6: The contours of the pressure perturbation and the velocity $\left(\sqrt{u^{2}+v^{2}}\right)$ of the small amplitude wave propagation problem in Section 5.3 by non-well-balanced WENO scheme at time $t=0.2$ with $200 \times 200$ cells. Left: pressure perturbation. Right: velocity.
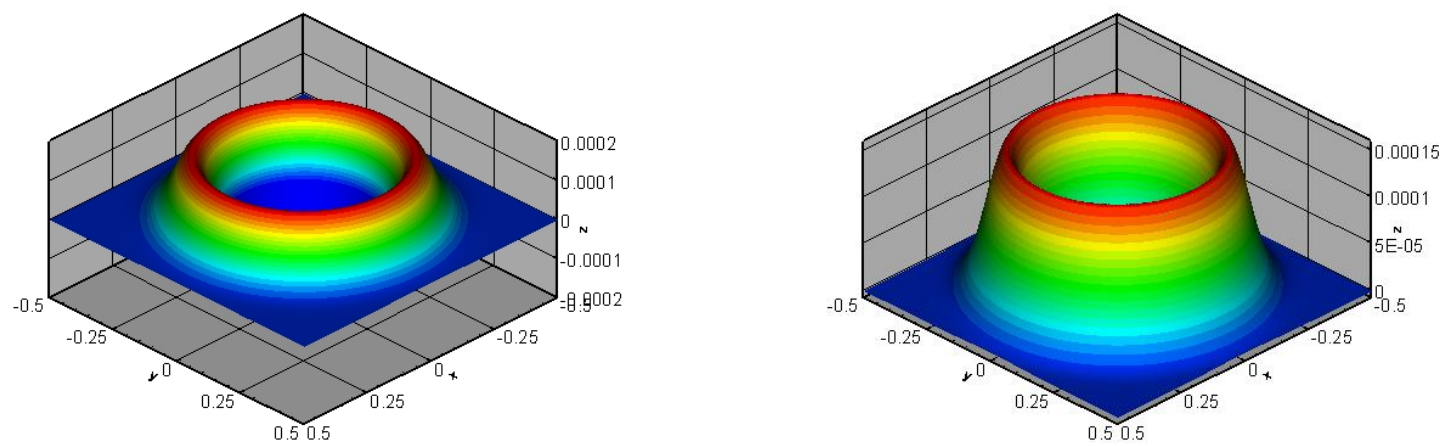

Fig. 5.7: The 3D views of the pressure perturbation and the velocity $\left(\sqrt{u^{2}+v^{2}}\right)$ of the small amplitude wave propagation problem in Section 5.3 by well-balanced WENO scheme at time $t=0.2$ with $200 \times 200$ cells. Left: pressure perturbation. Right: velocity. 

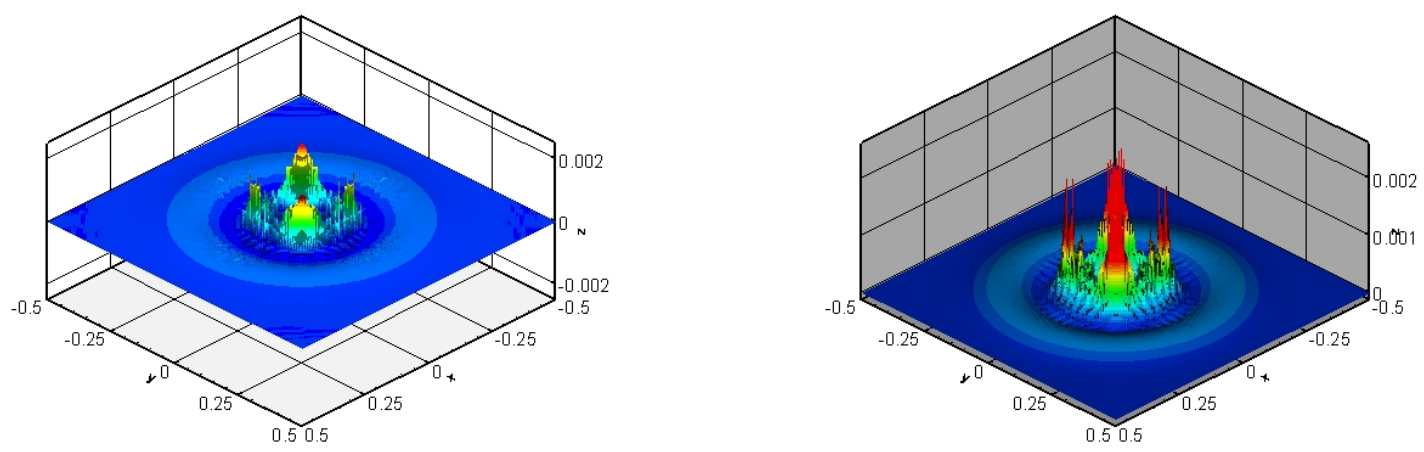

Fig. 5.8: The 3D views of the pressure perturbation and the velocity $\left(\sqrt{u^{2}+v^{2}}\right)$ of the small amplitude wave propagation problem in Section 5.3 by non-well-balanced WENO scheme at time $t=0.2$ with $200 \times 200$ cells. Left: pressure perturbation. Right: velocity.

Rigorous analysis as well as extensive numerical experiments all suggest that our proposed WENO schemes maintain the well-balanced property for both the isothermal and polytropic equilibriums solutions, and are able to capture the small perturbations of these hydrostatic balances states well on relatively coarse meshes.

\section{Acknowledgements}

The research of the first author is supported by the Natural Science Foundation of P.R. China (No. 11201254; 11401332) and the Project for Scientific Plan of Higher Education in Shandong Providence of P.R. China (No. J12LI08). This work was partially performed at the State Key Laboratory of Science/Engineering Computing of P.R. China by virtue of the computational resources of Professor Li Yuan's group. The first author is also thankful to Professor Li Yuan for his kind invitation. The research of the second author is partially supported by NSF grant DMS-1216454. 


\section{References}

[1] E. Audusse, F. Bouchut, M.-O. Bristeau, R. Klein, and B. Perthame. A fast and stable well-balanced scheme with hydrostatic reconstruction for shallow water flows. SIAM Journal on Scientific Computing, 25:2050-2065, 2004.

[2] A. Bermudez and M. E. Vazquez. Upwind methods for hyperbolic conservation laws with source terms. Computers and Fluids, 23:1049-1071, 1994.

[3] N. Botta, R. Klein, S. Langenberg, and S. Lützenkirchen. Well-balanced finite volume methods for nearly hydrostatic flows. Journal of Computational Physics, 196:539-565, 2004.

[4] P. Chandrashekar and C. Klingenberg. A second order well-balanced finite volume scheme for Euler equations with gravity. SIAM Journal on Scientific Computing, 37:382?02, 2015.

[5] A. Chertock, S. Cui, A. Kurganovz, S. N. Özcan, and E. Tadmor. Well-balanced centralupwind schemes for the Euler equations with gravitation. SIAM Journal on Scientific Computing, submitted.

[6] D. Ghosh and E. M. Constantinescu. A well-balanced, conservative finite difference algorithm for atmospheric flows. AIAA Journal, in press.

[7] J. M. Greenberg and A. Y. LeRoux. A well-balanced scheme for the numerical processing of source terms in hyperbolic equations. SIAM Journal on Numerical Analysis, 33:1-16, 1996.

[8] G. Jiang and C.-W. Shu. Efficient implementation of weighted ENO schemes. Journal of Computational Physics, 126:202-228, 1996.

[9] R. Käppeli and S. Mishra. Well-balanced schemes for the Euler equations with gravitation. Journal of Computational Physics, 259:199 - 219, 2014. 
[10] R. Käppeli and S. Mishra. A well-balanced finite volume scheme for the Euler equations with gravitation. The exact preservation of hydrostatic equilibrium with arbitrary entropy stratification Astronomy 8 Astrophysics, 587, A94, 2016.

[11] R. J. LeVeque. Balancing source terms and flux gradients on high-resolution Godunov methods: the quasi-steady wave-propagation algorithm. Journal of Computational Physics, 146:346-365, 1998.

[12] R. J. LeVeque and D. S. Bale. Wave propagation methods for conservation laws with source terms. In Proceedings of the 7th International Conference on Hyperbolic Problems, pages 609-618, 1998.

[13] G. Li and Y. Xing. Well-balanced discontinuous Galerkin methods for the Euler equations under gravitational fields. Journal of Scientific Computing, in press.

[14] J. Luo, K. Xu, and N. Liu. A well-balanced symplecticity-preserving gas-kinetic scheme for hydrodynamic equations under gravitational field. SIAM Journal on Scientific Computing, 33:2356-2381, 2011.

[15] B. Perthame and C. Simeoni. A kinetic scheme for the Saint-Venant system with a source term. Calcolo, 38:201-231, 2001.

[16] C.-W. Shu. Essentially non-oscillatory and weighted essentially non-oscillatory schemes for hyperbolic conservation laws. In A. Quarteroni, editor, Advanced Numerical Approximation of Nonlinear Hyperbolic Equations, pages 325-432. Lecture Notes in Mathematics, volume 1697, Springer, 1998.

[17] C.-W. Shu and S. Osher. Efficient implementation of essentially non-oscillatory shockcapturing schemes. Journal of Computational Physics, 77:439-471, 1988.

[18] A. Slyz and K. H. Prendergast. time independent gravitational fields in the BGK scheme for hydrodynamics. Astronomy \& Astrophysics Supplement Series, 139:199-217, 1999. 
[19] C.T. Tian, K. Xu, K.L. Chan, and L.C. Deng. A three-dimensional multidimensional gas-kinetic scheme for the navier-stokes equations under gravitational fields. Journal of Computational Physics, 226:2003-2027, 2007.

[20] Y. Xing and C.-W. Shu. High order finite difference WENO schemes with the exact conservation property for the shallow water equations. Journal of Computational Physics, 208:206-227, 2005.

[21] Y. Xing and C.-W. Shu. A new approach of high order well-balanced finite volume WENO schemes and discontinuous Galerkin methods for a class of hyperbolic systems with source terms. Communications in Computational Physics, 1:100-134, 2006.

[22] Y. Xing and C.-W. Shu. High order well-balanced WENO scheme for the gas dynamics equations under gravitational fields. Journal of Scientific Computing, 54:645-662, 2013.

[23] Y. Xing and C.-W. Shu. A survey of high order schemes for the shallow water equations. Journal of Mathematical Study, 47:221-249, 2014.

[24] Y. Xing, X. Zhang, and C.-W. Shu. Positivity-preserving high order well-balanced discontinuous Galerkin methods for the shallow water equations. Advances in Water Resources, 33:1476-1493, 2010.

[25] K. Xu. A well-balanced gas-kinetic scheme for the shallow-water equations with source terms. Journal of Computational Physics, 178:533-562, 2002.

[26] K. Xu, J. Luo, and S. Chen. A well-balanced kinetic scheme for gas dynamic equations under gravitational field. Advances in Applied Mathematics and Mechanics, 2:200-210, 2010. 